\title{
Sign(al-to-noise) Problems in Lattice Gauge Theory
}

FERMILAB-SLIDES-21-032-T

\section{Michael Wagman}

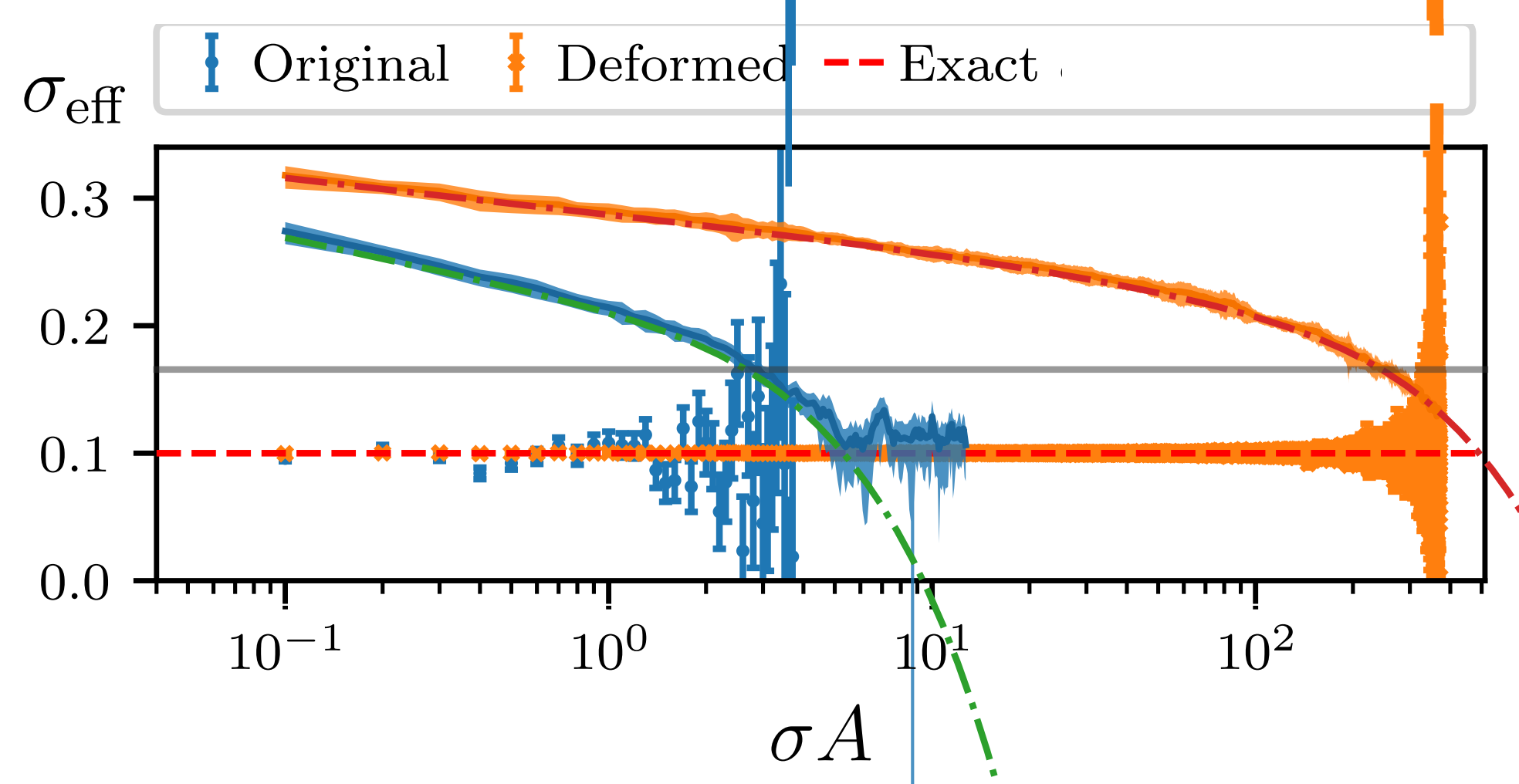

\section{University of lowa Nuclear and Particle Physics Seminar}




\section{Solving the Standard Model}

Simple quantities like the protonneutron mass difference have been computed with percentlevel precision in lattice QCD+QED
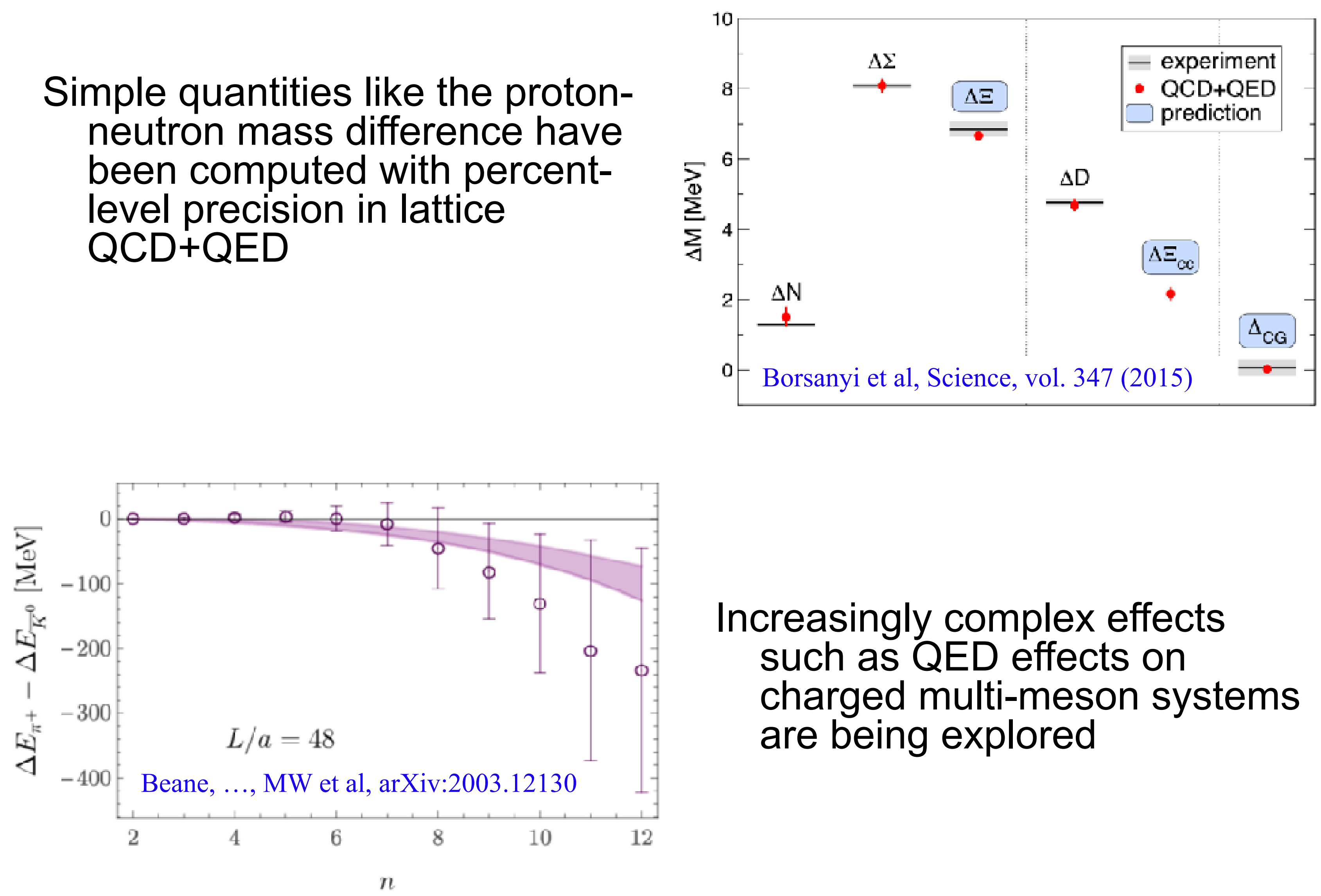

Increasingly complex effects such as QED effects on charged multi-meson systems are being explored 


\section{Nuclear matrix elements from LQCD}

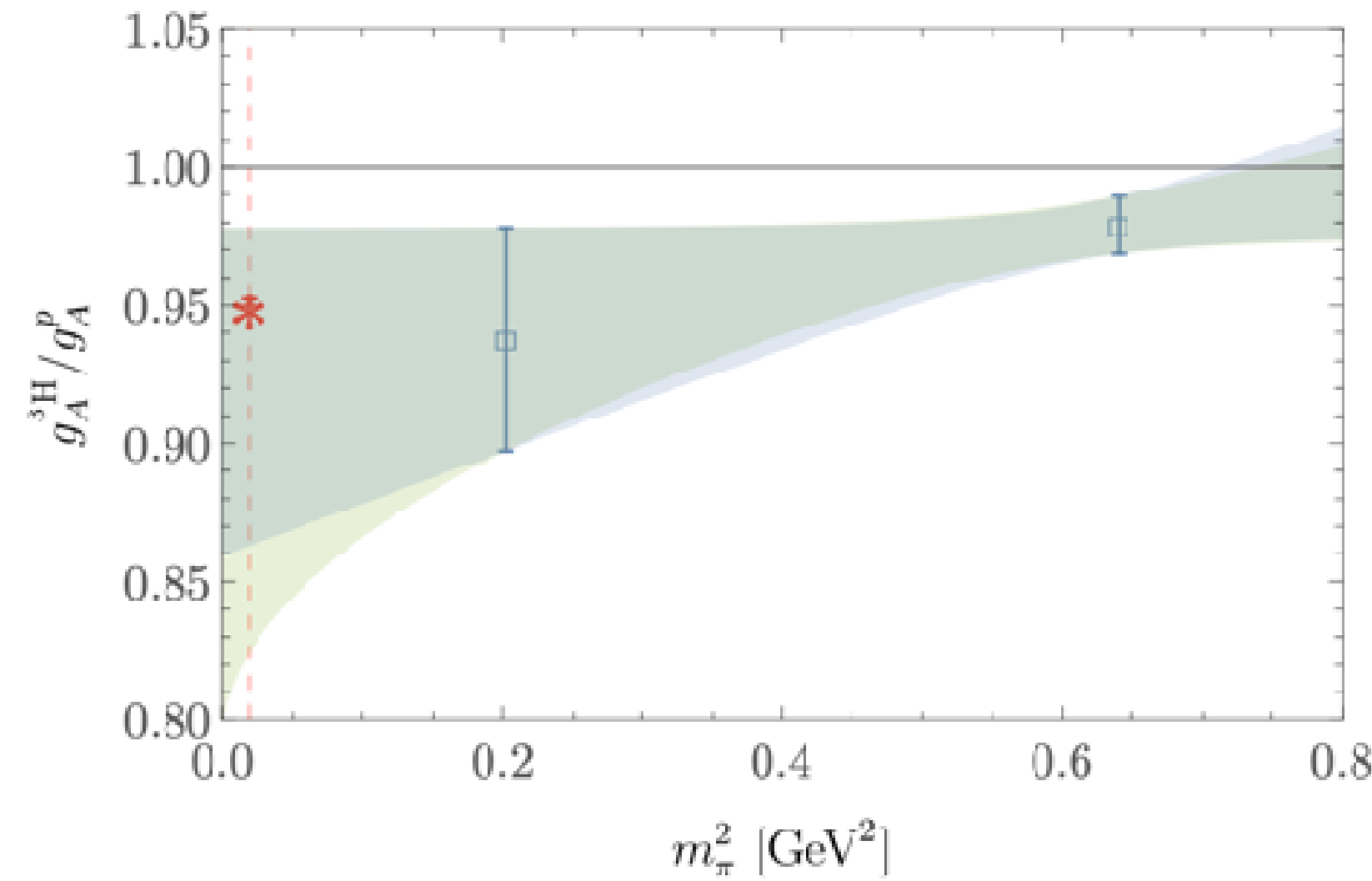

Parreño, ..., MW, et al [NPLQCD] arXiv:2102.xx

The $u-d$ quark momentum fractions of light nuclei have been computed in exploratory calculations at heavier-than-physical quark masses

\author{
Triton beta-decay rate \\ computed from lattice QCD, \\ effects of axial two-body \\ currents visible
}

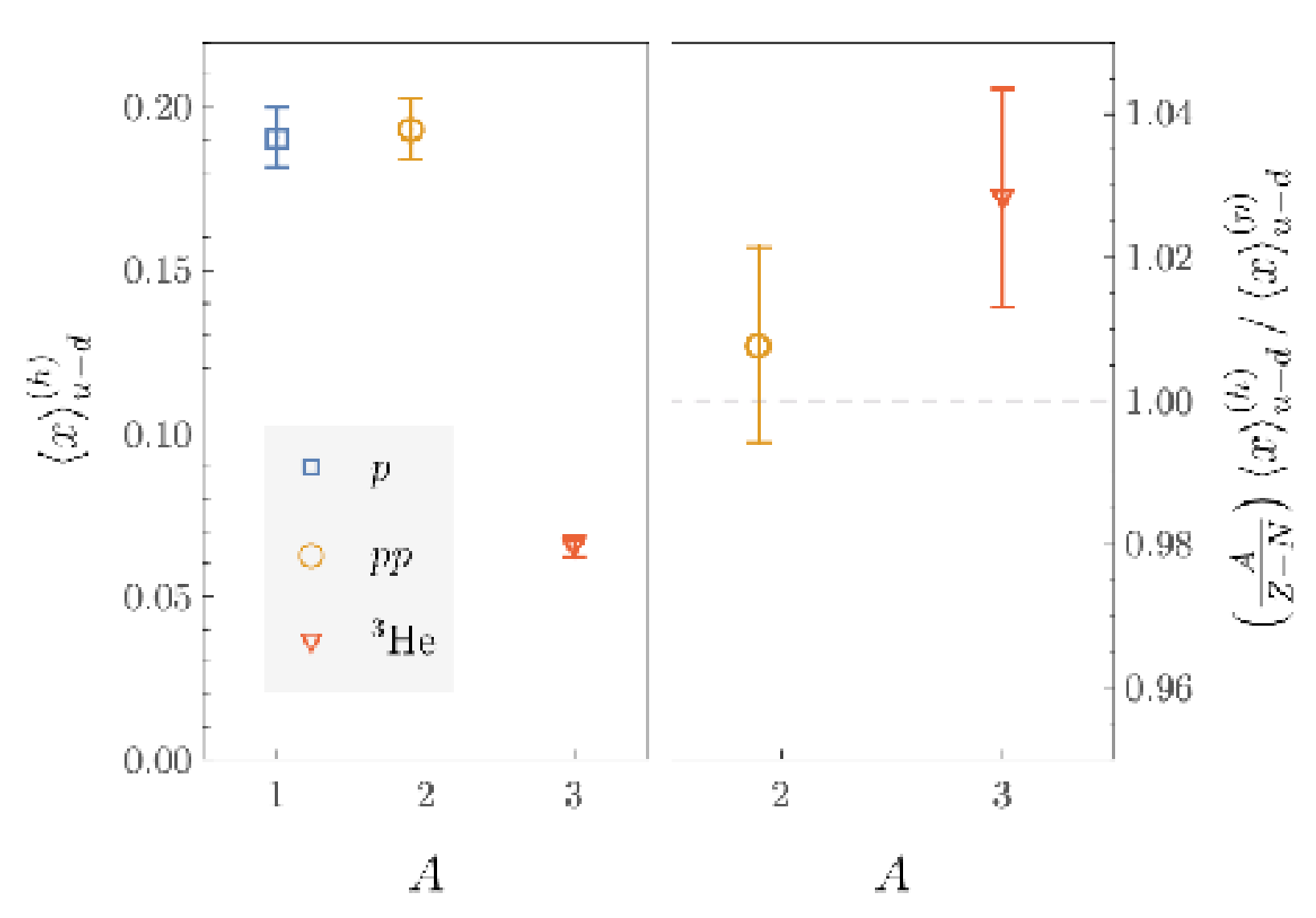

Detmold, ..., MW, et al [NPLQCD], arXiv:2101.12668 


\section{Excited-state effects}

Euclidean correlation functions have spectral representation

$$
\begin{aligned}
G(\tau) & =\langle N(\tau) \bar{N}(0)\rangle=\sum_{n}\langle 0|N| n\rangle e^{-E_{n} \tau}\langle n|\bar{N}| 0\rangle \\
& \equiv \sum_{n} Z_{n} e^{-E_{n} \tau}
\end{aligned}
$$

Precise studies of multi-nucleon systems face challenges from unbound finite-volume excited states

$$
\vec{p}=\frac{2 \pi \vec{n}}{L} \quad E_{N N} \approx 2 \sqrt{M_{N}^{2}+\left(\frac{2 \pi n}{L}\right)^{2}}
$$

Analogous issues with $N \pi$ excited-states may impact axial form factor calculations

Better tools for needed for dealing with multi-hadron excited states 


\section{The signal-to-noise problem}

Monte Carlo noise in nucleon/nuclear correlation functions grows exponentially with Euclidean time separation

Correlation function variance can be analyzed as a correlation function

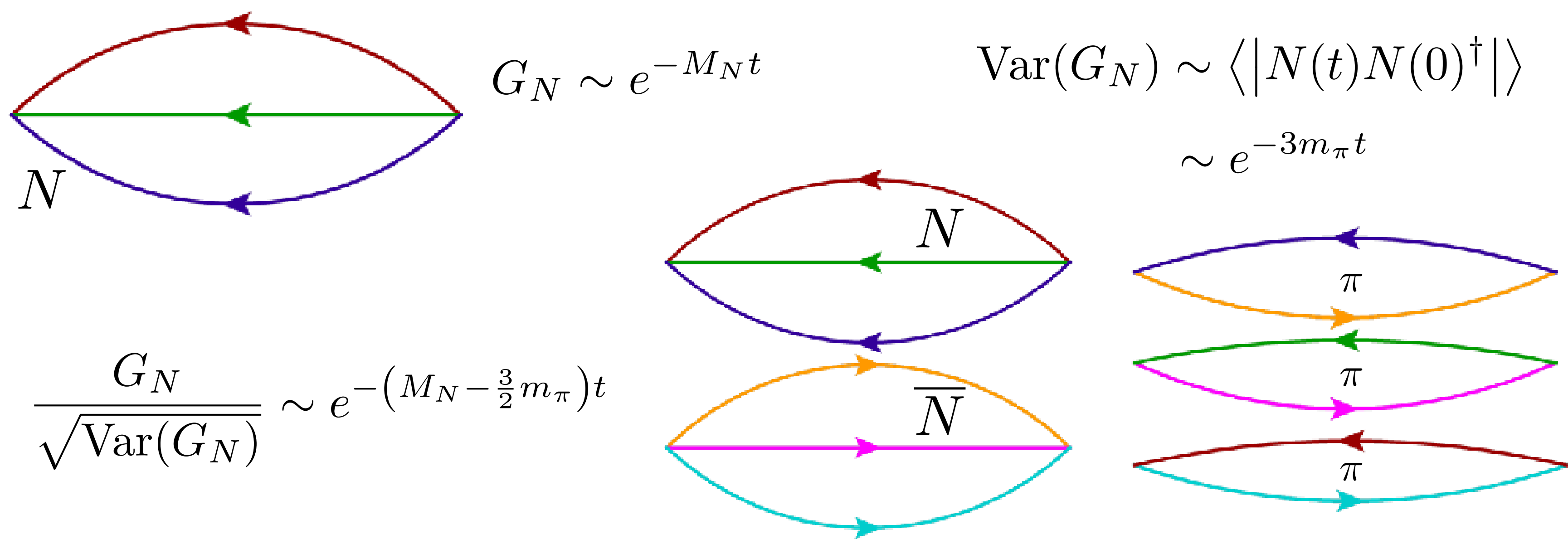

Multi-nucleon: $\sim e^{-A\left(M_{N}-\frac{3}{2} m_{\pi}\right) t}$

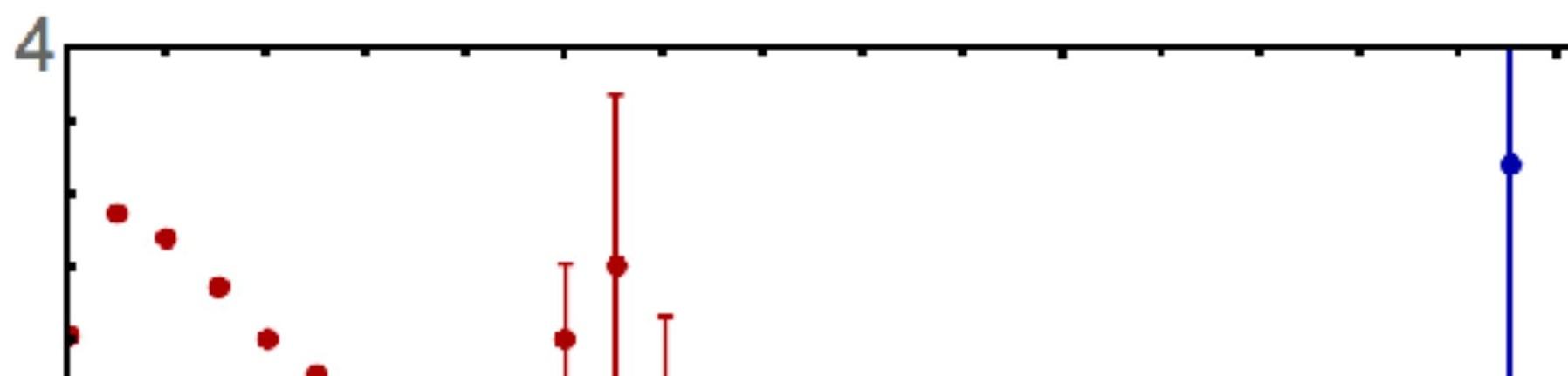




\section{The sign(al-to-noise) problem}

Nucleon correlation functions are real on average but complex in generic gauge field backgrounds

$$
G_{N}(t)=\left\langle e^{R(t)+i \theta(t)}\right\rangle
$$

Contributions to the energy from phase fluctuations lead to StN problems

$$
\operatorname{StN}\left(e^{i \theta(t)}\right) \sim \frac{\left\langle e^{i \theta(t)}\right\rangle}{\left\langle\left|e^{i \theta(t)}\right|^{2}\right\rangle}=\left\langle e^{i \theta(t)}\right\rangle \sim e^{-m_{\theta} t}
$$

Empirically, phase fluctuations ("sign problem") reproduce the ParisiLepage signal-to-noise problem

$$
m_{R}(t)=\ln \left(\frac{\left\langle e^{R(t)}\right\rangle}{\left\langle e^{R(t+1)}\right\rangle}\right) \sim \frac{3}{2} m_{\pi}
$$

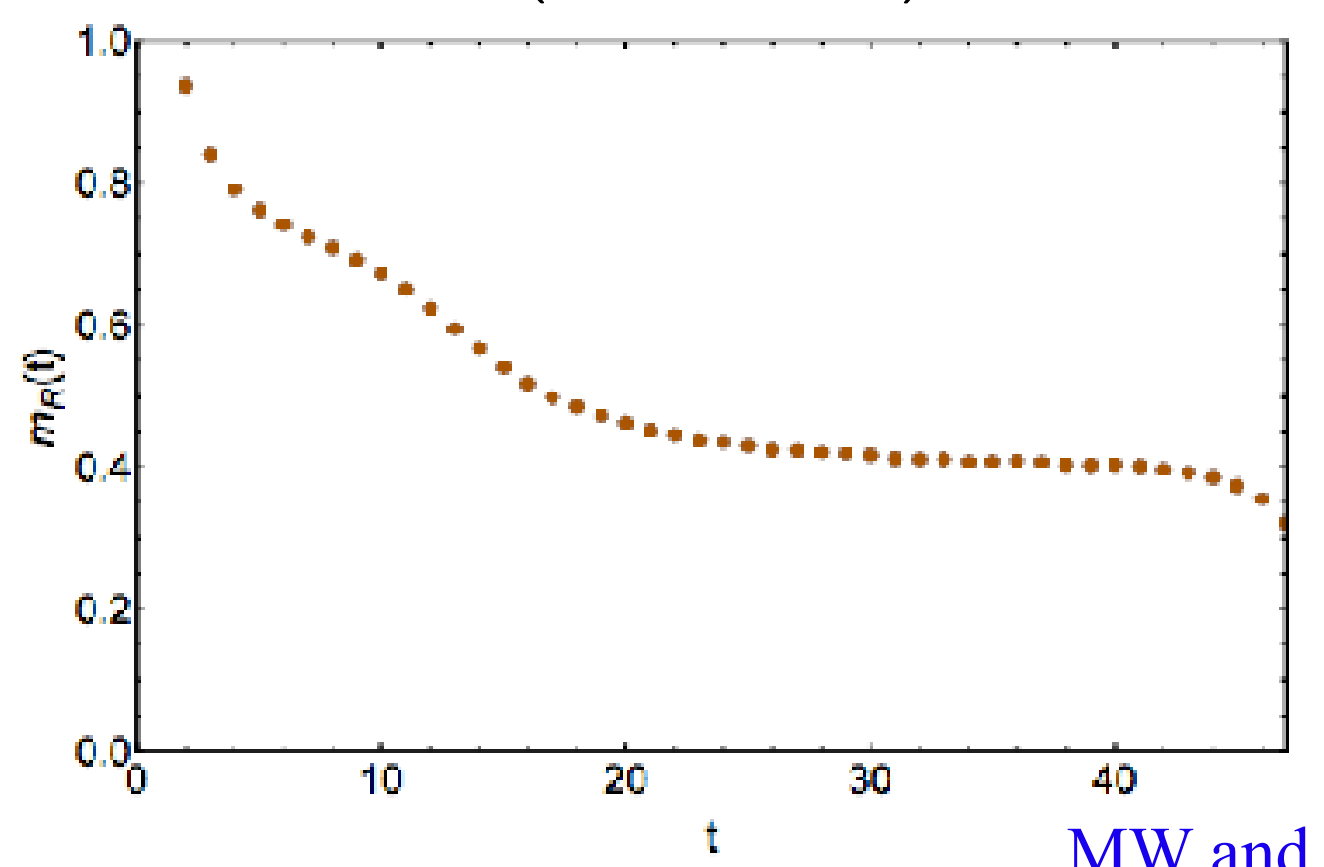

$$
m_{\theta}(t)=\ln \left(\frac{\left\langle e^{i \theta(t)}\right\rangle}{\left\langle e^{i \theta(t+1)}\right\rangle}\right) \sim M_{N}-\frac{3}{2} m_{\pi}
$$

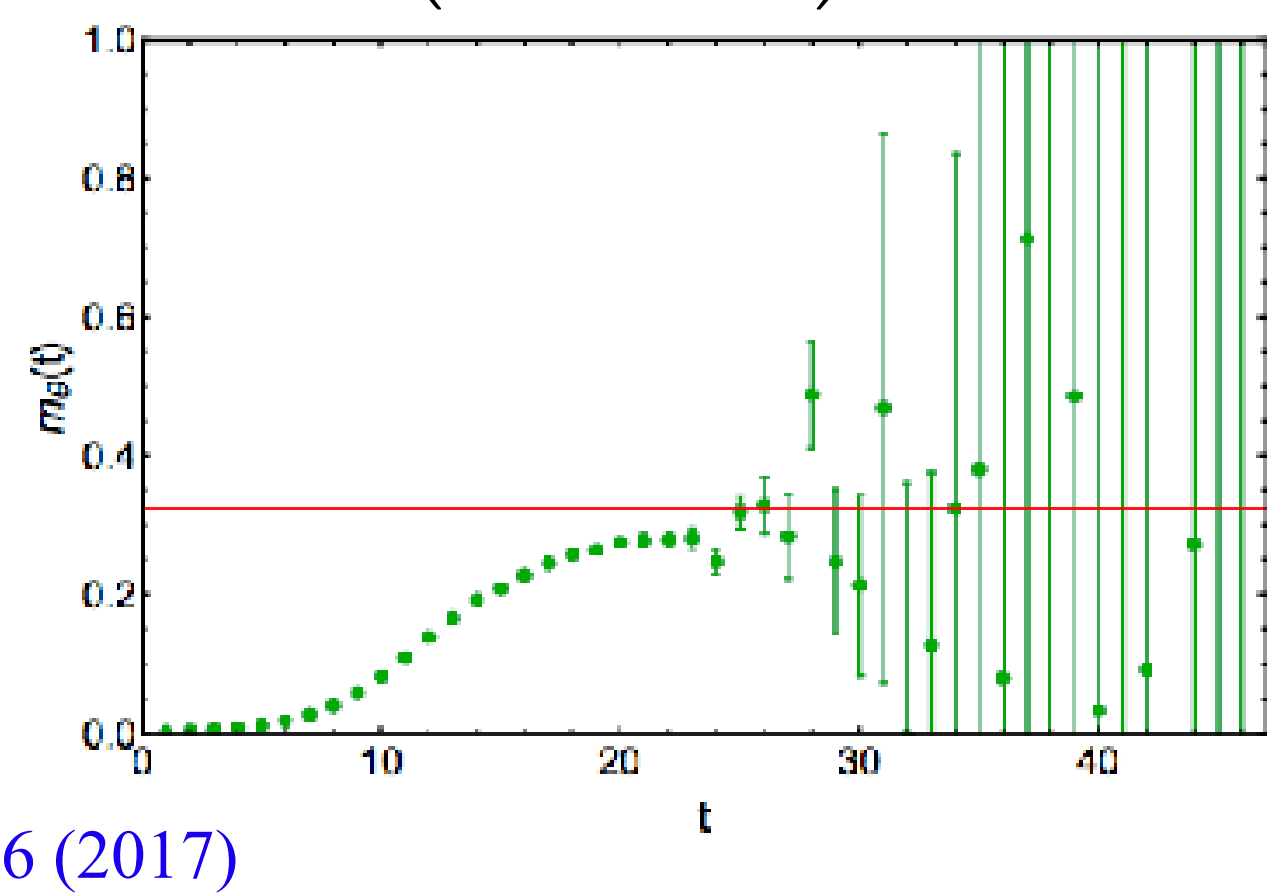




\section{A nice toy sign problem}

$\int_{-\pi}^{\pi} \frac{d \phi}{2 \pi} e^{i \phi} e^{\beta \cos (\phi)}=I_{1}(\beta)=\left\langle e^{i \phi}\right\rangle_{\beta} I_{0}(\beta)$

$\operatorname{Var}_{\beta}\left[\operatorname{Re} e^{i \phi}\right]=\left\langle\cos ^{2}(\phi)\right\rangle_{\beta}-\left\langle e^{i \phi}\right\rangle_{\beta}^{2}$

$=\frac{1}{2}\left[1+\frac{I_{2}(\beta)}{I_{0}(\beta)}\right]-\left[\frac{I_{1}(\beta)}{I_{0}(\beta)}\right]^{2}$

For uniform phase

fluctuations, average phase vanishes but variance is always $O(1)$ 


\section{Contour deformations}

$$
\int_{-\pi}^{\pi} \frac{d \phi}{2 \pi} e^{i \phi} e^{\beta \cos (\phi)}=I_{1}(\beta)=\left\langle e^{i \phi}\right\rangle_{\beta} I_{0}(\beta)
$$

Stokes' theorem + holomorphic integrand integral result unaffected by contour deormation

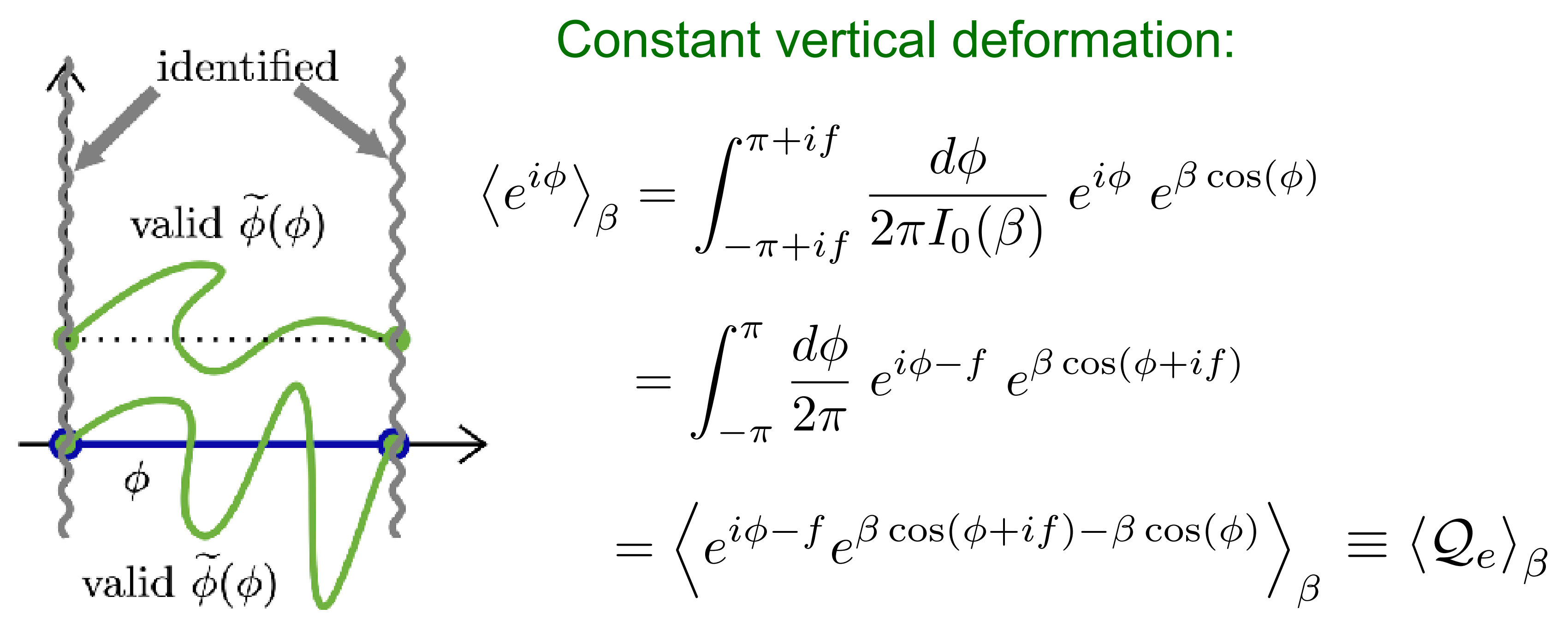




\section{Variance reduction}

The variance involves non-holomorphic integrands

$\operatorname{Var}_{\beta}\left[\operatorname{Re} \mathcal{Q}_{e}\right]=\left\langle\left(\operatorname{Re} \mathcal{Q}_{e}\right)^{2}\right\rangle_{\beta}-\left\langle e^{i \phi}\right\rangle_{\beta}^{2} \neq \operatorname{Var}_{\beta}\left[\operatorname{Re} e^{i \phi}\right]$

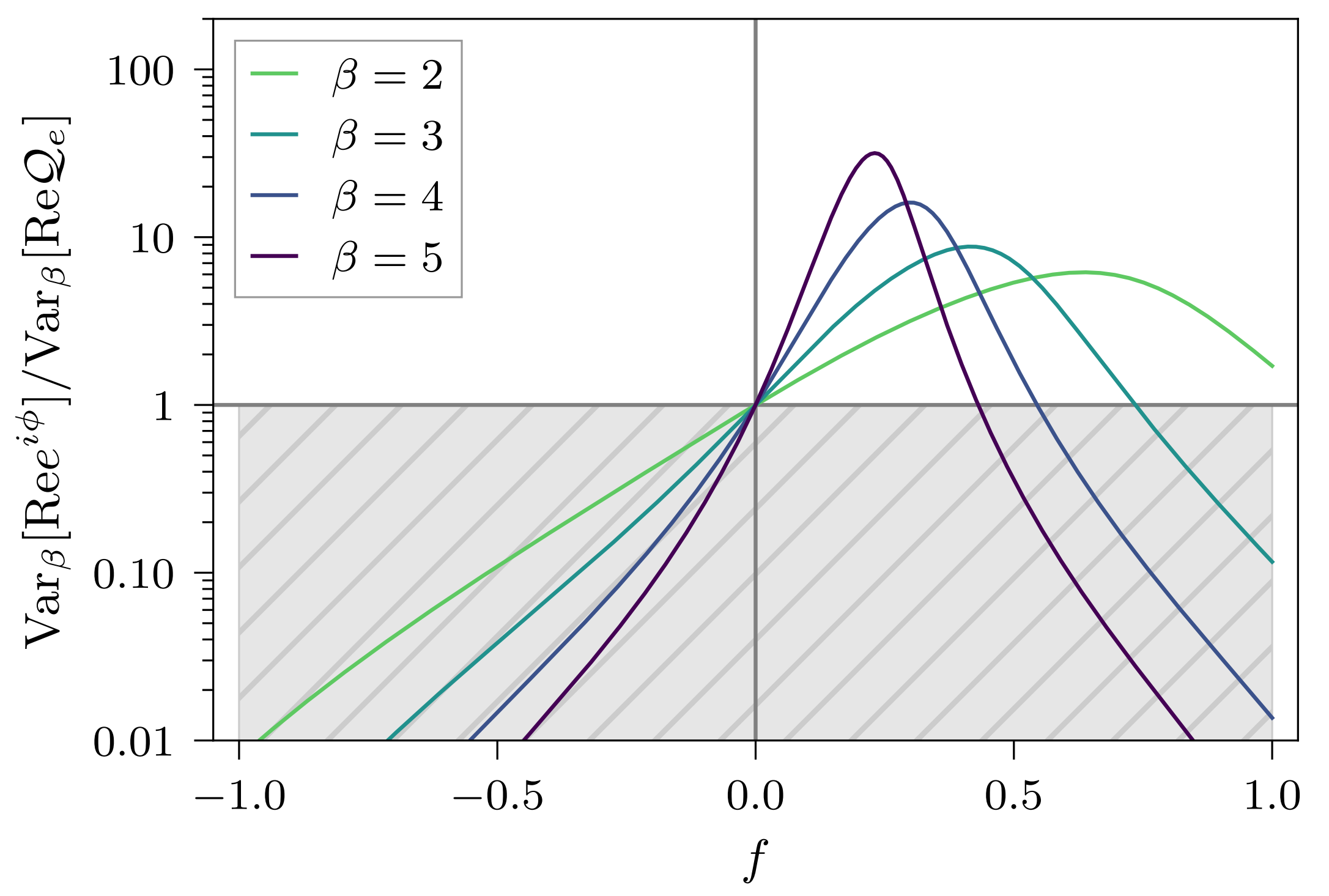

$\mathcal{Q}_{e}=e^{-f} e^{i \phi} e^{\Delta S}$ 


\section{Path integral contour deformations}

Consider a path integral with a sign problem

$$
\langle\mathcal{O}\rangle_{M}=\frac{1}{Z_{M}} \int_{\mathcal{M}} \mathcal{D} U e^{i S_{M}(U)} \mathcal{O}(U)
$$

Witten, AMS/IP

Stud.Adv.Math. 50 (2011)

Cristoforetti, Di Renzo, Scorzato, PRD 86 (2012)

\section{Review:}

Alexandru, Basar, Bedaque, Warrington, arXiv:2007.05436

Deform the integration contour

$$
\begin{aligned}
& =\frac{1}{Z_{M}} \int_{\widetilde{\mathcal{M}}} \mathcal{D} U e^{i S_{M}(\widetilde{U})} \mathcal{O}(\widetilde{U}) \\
& =\frac{1}{Z_{M}} \int_{\mathcal{M}} \mathcal{D} U J(U) e^{i S_{M}(\widetilde{U}(U))} \mathcal{O}(\widetilde{U}(U))
\end{aligned}
$$

Deformed integrand can have less severe sign problem

$$
\begin{gathered}
=\frac{1}{Z_{M}} \int_{\mathcal{M}} \mathcal{D} U|J(U)| e^{-\operatorname{Im}\left[S_{M}(\widetilde{U}(U))\right]} \mathcal{O}(\widetilde{U}(U)) \\
\quad \times e^{i \operatorname{Re}\left[S_{M}(\widetilde{U}(U))\right]+i \arg [J(U)]}
\end{gathered}
$$




\section{Observifolds}

Deformed observables method: contour deformations without modifying Monte Carlo sampling

$$
\begin{aligned}
& \langle\mathcal{O}\rangle=\frac{1}{Z} \int_{\mathcal{M}} \mathcal{D} U e^{-S(U)} \mathcal{O}(U) \\
& =\frac{1}{Z} \int_{\widetilde{\mathcal{M}}} \mathcal{D} \widetilde{U} e^{-S(\widetilde{U})} \mathcal{O}(\widetilde{U}) \\
& =\frac{1}{Z} \int_{\mathcal{M}} \mathcal{D} U e^{-S(U)} \operatorname{det}\left(\frac{\partial \widetilde{U}}{\partial U}\right) e^{-S(\widetilde{U}(U))+S(U)} \mathcal{O}(\widetilde{U}(U)) \\
& \equiv \frac{1}{Z} \int_{\mathcal{M}} \mathcal{D} U e^{-S(U)} \mathcal{Q}(U) \\
& \begin{aligned}
\langle\mathcal{O}\rangle & =\langle\mathcal{Q}\rangle \\
\operatorname{Var}[\mathcal{O}] & \neq \operatorname{Var}[\mathcal{Q}]
\end{aligned} \\
& \begin{aligned}
\langle\mathcal{O}\rangle & =\langle\mathcal{Q}\rangle \\
\operatorname{Var}[\mathcal{O}] & \neq \operatorname{Var}[\mathcal{Q}]
\end{aligned}
\end{aligned}
$$




\section{Lattice gauge theory}

Gauge transformations act on matter fields as

$$
\begin{aligned}
& \psi_{x}^{a} \rightarrow \Omega_{x}^{a b} \psi_{x}^{b} \\
& \Omega_{x} \in S U(N), U(1)
\end{aligned}
$$

Gauge field acts as parallel transporter in color space

$$
\begin{aligned}
& D_{\mu}^{a b} \psi_{x}^{b}=U_{x, \mu}^{a b} \psi_{x+\hat{\mu}}^{b}-\psi_{x}^{a} \\
& D_{\mu} \psi_{x}^{a} \rightarrow \Omega_{x}^{a b} D_{\mu} \psi_{x}^{b}
\end{aligned}
$$

$$
\begin{gathered}
U_{x, \mu} \in S U(N), U(1) \\
U_{x, \mu}=e^{i \int_{x}^{x+\hat{\mu}} d y A_{\mu}(y)} \\
U_{x, \mu} \rightarrow \Omega_{x} U_{x, \mu} \Omega_{x+\hat{\mu}}^{\dagger}
\end{gathered}
$$

Gauge invariant building blocks:
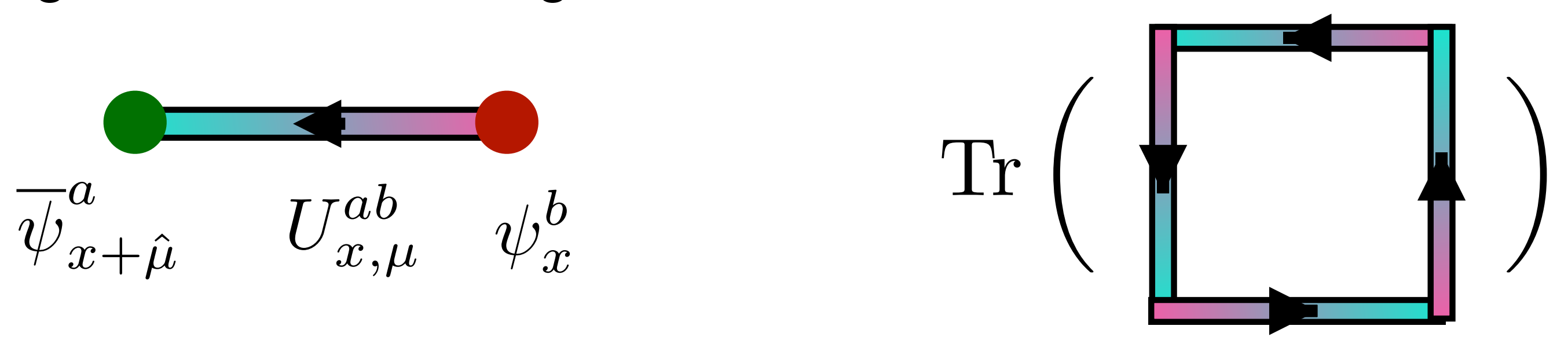


\section{Wilson loops}

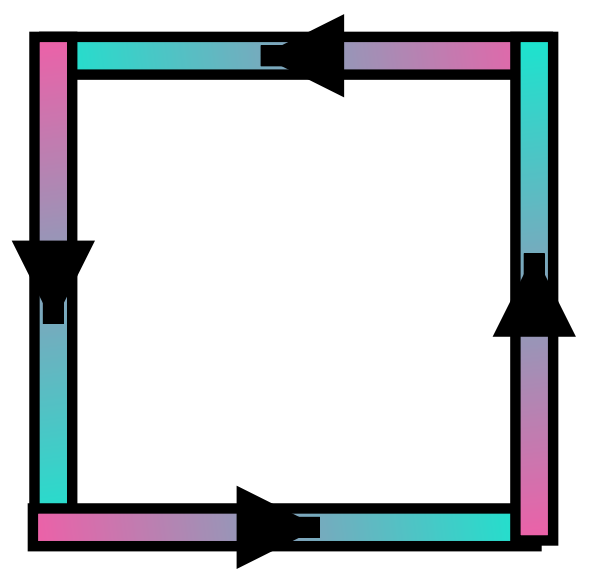

$$
W_{\mathcal{A}}=\prod_{x, \mu \in \partial \mathcal{A}} U_{x, \mu}=
$$
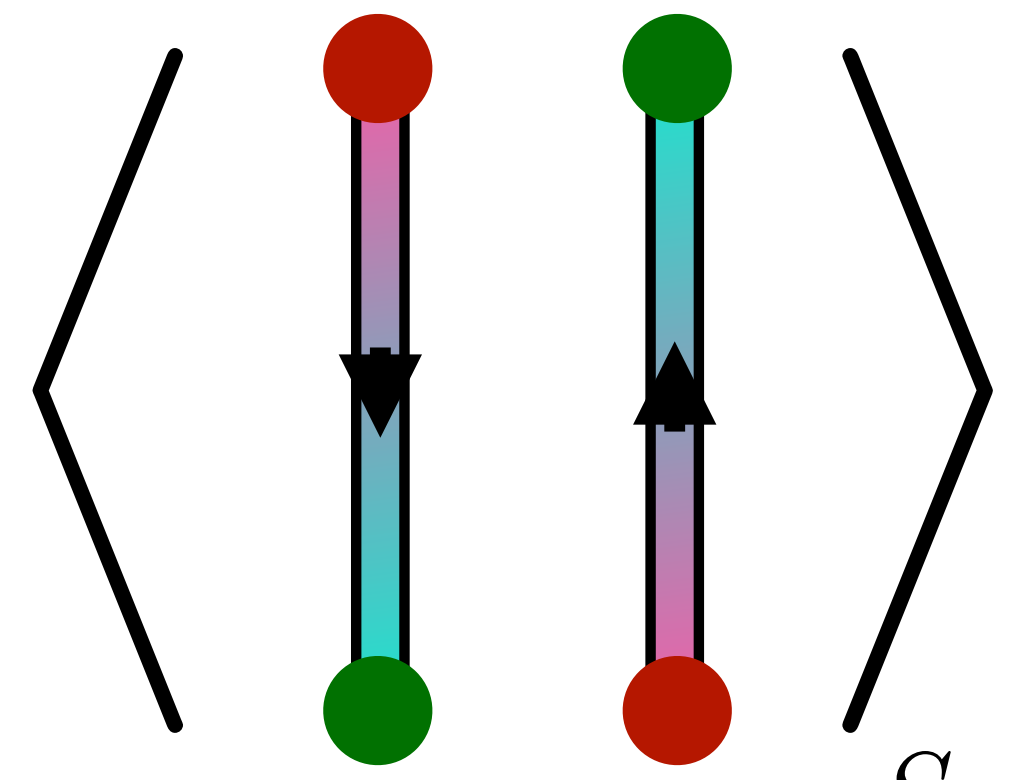

Wilson loops are equivalent to static quark propagators

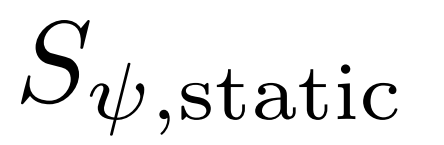

$$
\text { Since by equations of motion } \psi_{(\vec{x}, \tau)}=\prod_{\tau^{\prime}=0} U_{\left(\vec{x}, \tau^{\prime}\right), 4}^{-1} \psi_{(\vec{x}, 0)}
$$

Static quark potential accessible from Wilson loops

$$
\left\langle W_{r \times \tau}\right\rangle=\sum_{n} Z_{n} e^{-E_{n}(r) \tau}=e^{-V(r) \tau}+\ldots
$$




\section{Plaquettes}

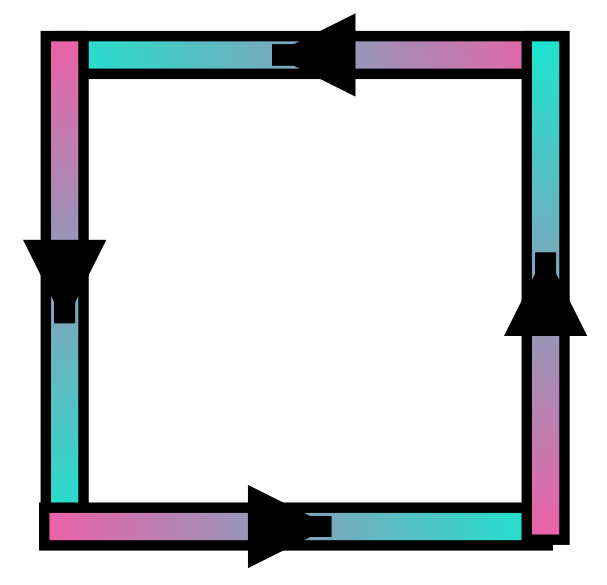

"Plaquettes" are 1x1 Wilson loops

$$
P_{x, \mu \nu}=U_{x, \mu} U_{x+\hat{\mu}, \nu} U_{x+\hat{\mu}+\hat{n u}, \mu}^{-1} U_{x+\hat{\nu}, \nu}^{-1}
$$

Wilson action provides simple, gauge-invariant action with correct naive continuum limit

$$
S_{W}(U)=\frac{1}{g^{2}} \sum_{x} \sum_{\mu<\nu} \operatorname{Tr}\left[2-P_{x, \mu \nu}-P_{x, \mu \nu}^{-1}\right]
$$

Wilson loops can be expressed using plaquettes, most simply taking open boundary conditions and gauge-fixing $U_{x, \nu}=1$

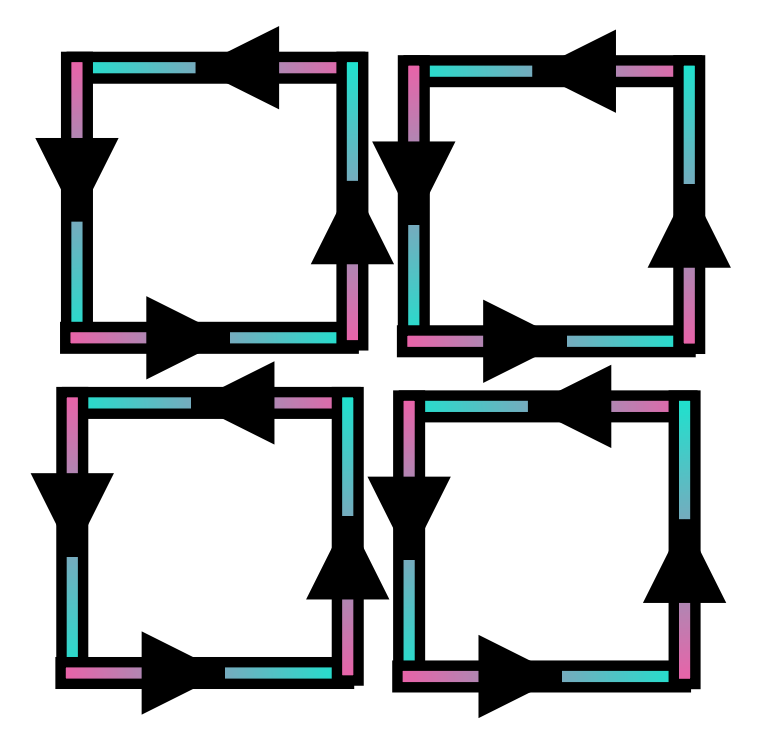

$$
W_{\mathcal{A}}=\prod_{x, \mu \in \partial \mathcal{A}} U_{x, \mu}=\prod_{x \in \mathcal{A}} P_{x, \mu \nu}
$$




\section{D Confinement}

In 2D, Wilson loop expectation values further factorize into products of single-plaquette expectation values

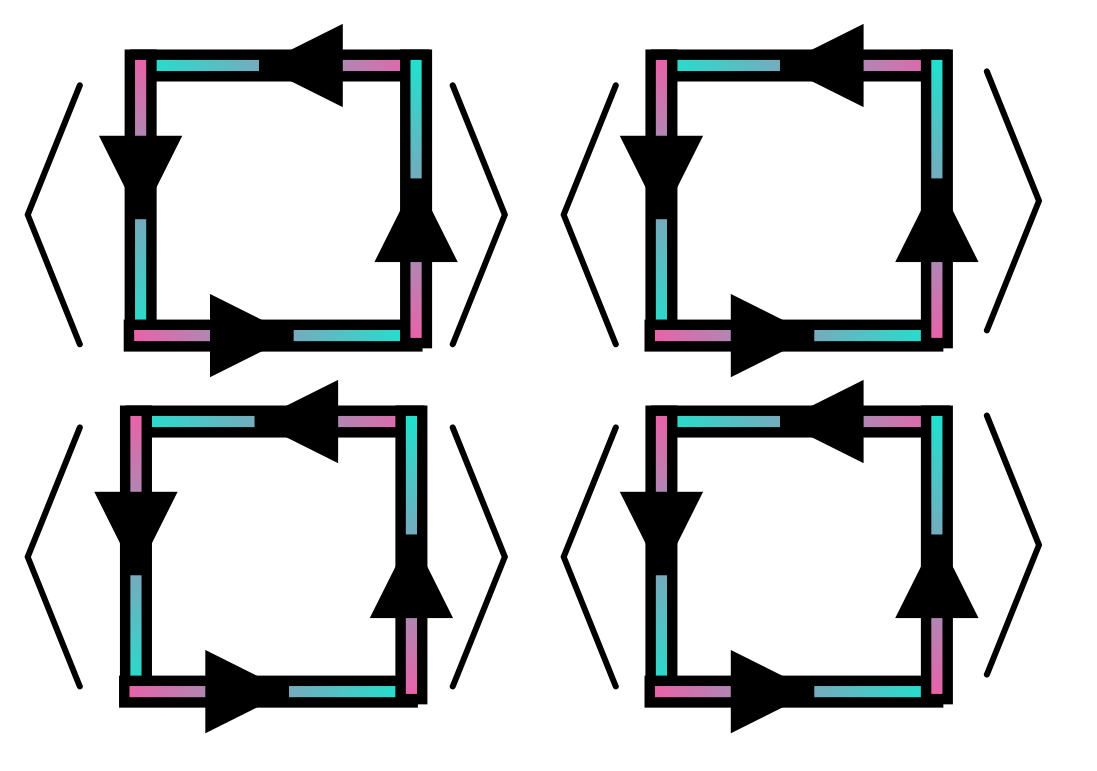

$$
\left\langle\operatorname{Tr}\left(W_{\mathcal{A}}\right)\right\rangle=\prod_{x \in \mathcal{A}}\left\langle\operatorname{Tr}\left(P_{x}\right)\right\rangle=\langle\operatorname{Tr}(P)\rangle^{A}
$$

Implies confinement, static quark potential

$$
V(r)=\sigma r
$$

String tension:

$$
\sigma=-\ln \left\langle\frac{1}{N} \operatorname{Tr}(P)\right\rangle
$$

Confining potential arises for any gauge group in 2D from factorization

$$
\begin{gathered}
\sigma_{U(1)}=\ln \left(\frac{I_{0}\left(1 / e^{2}\right)}{I_{1}\left(1 / e^{2}\right)}\right) \\
\sigma_{S U(2)}=\ln \left(\frac{I_{1}\left(4 / g^{2}\right)}{I_{2}\left(4 / g^{2}\right)}\right)
\end{gathered}
$$




\section{D Wilson loop noise}

Squared traces also factorize, but differently
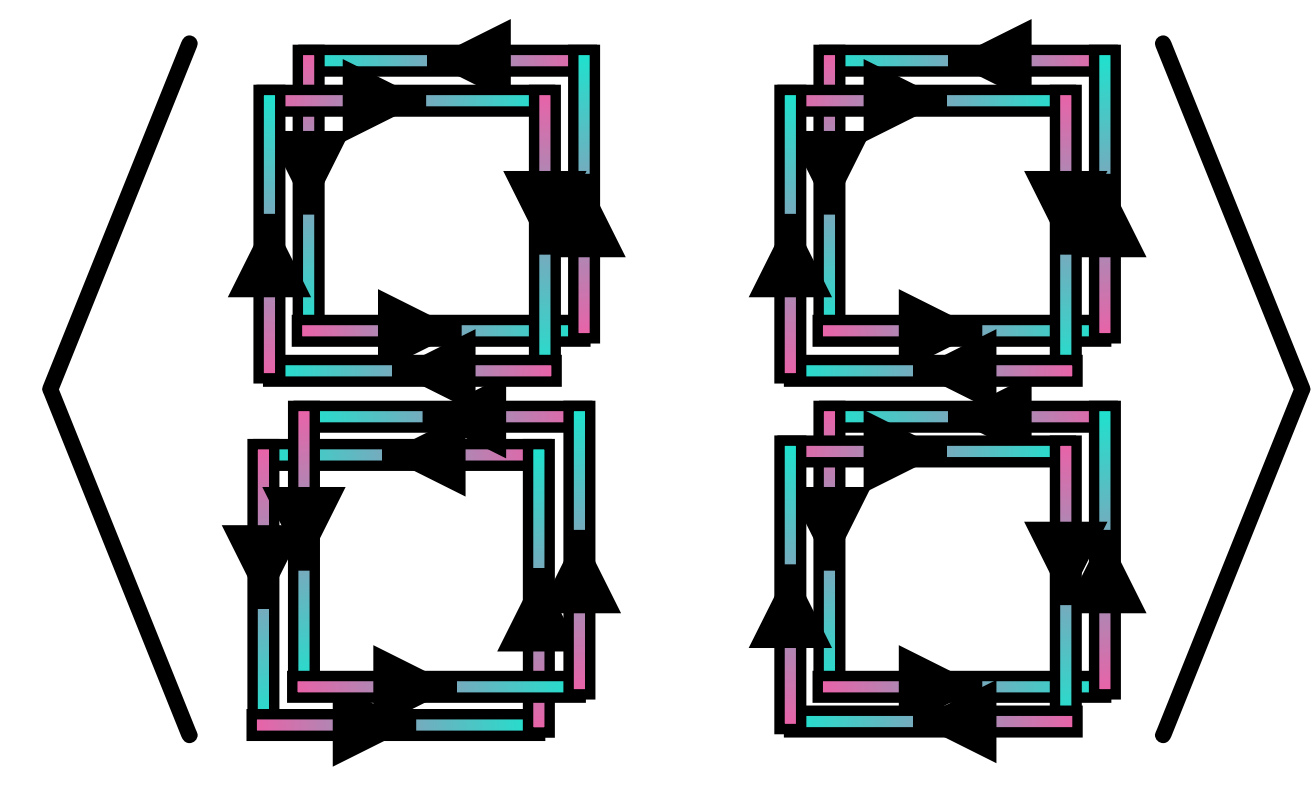

$$
\begin{aligned}
& \left\langle\left|\operatorname{Tr}\left(W_{\mathcal{A}}\right)^{2}\right|\right\rangle=1+\left(N^{2}-1\right)\left\langle\chi_{1,-1}\right\rangle^{A} \\
& \left\langle\operatorname{Tr}\left(W_{\mathcal{A}}\right)^{2}\right\rangle=\frac{N(N+1)}{2}\left\langle\chi_{2}\right\rangle^{A}+\frac{N(N-1)}{2}\left\langle\chi_{1,-1}\right\rangle^{A} \\
& \text { Detmold, Kanwar, Lamm, MW, Warrington, arXiv:2101.12668 }
\end{aligned}
$$

Phase (sign in $\mathrm{N}=2$ ) fluctuations of Wilson loop traces lead to exponential StN problem

$$
\begin{aligned}
& \operatorname{Var}\left[\frac{1}{N} \operatorname{Re} \operatorname{Tr}\left(W_{\mathcal{A}}\right)\right]=\frac{1}{2 N^{2}}+\ldots \\
& \operatorname{StN}\left[\frac{1}{N} \operatorname{Re} \operatorname{Tr}\left(W_{\mathcal{A}}\right)\right]=\sqrt{2} N n e^{-\sigma A}+\ldots
\end{aligned}
$$




\section{D U(1) contour deformations}

Using the parameterization

$$
P=e^{i \phi} \in U(1)
$$

$$
\left\langle W_{\mathcal{A}}\right\rangle=\left(\int \frac{d P}{2 \pi I_{0}\left(1 / e^{2}\right)} P e^{\frac{1}{2 e^{2}}\left(P+P^{-1}\right)}\right)^{A}
$$

$U(1)$ Wilson loops are products of toy sign problem integrals

$$
=\left(\int_{-\pi}^{\pi} \frac{d \phi}{2 \pi I_{0}\left(1 / e^{2}\right)} e^{i \phi} e^{\frac{1}{e^{2}} \cos (\phi)}\right)^{A}
$$

Contour deformation analogous to toy problem for $\mathrm{U}(1)$ Wilson loops

$$
e^{i \phi} \rightarrow e^{i(\phi+i f)}
$$
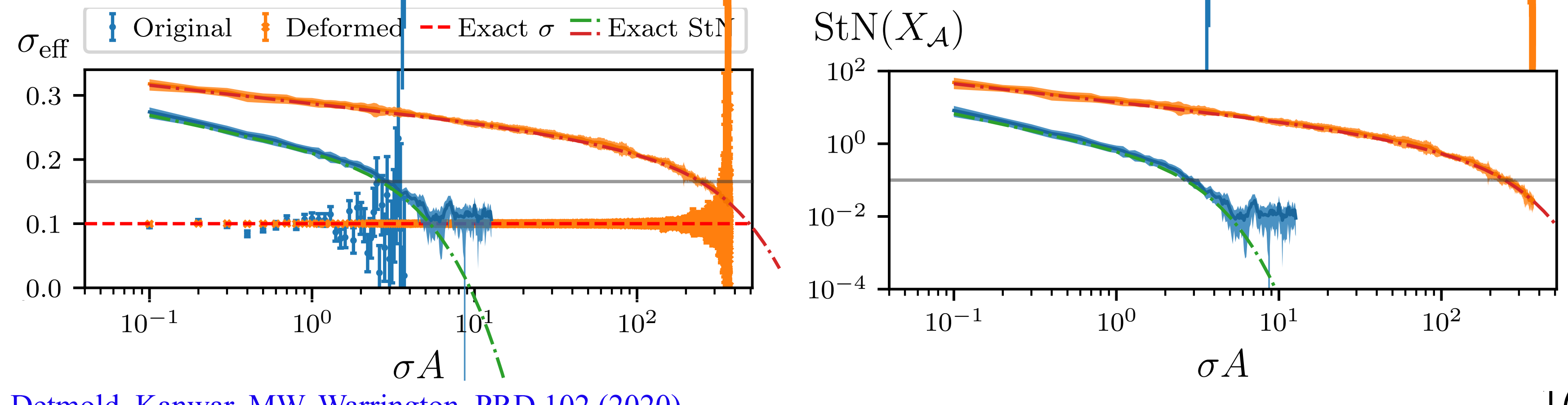

Detmold, Kanwar, MW, Warrington, PRD 102 (2020) 


\section{A mean toy sign problem}

An alternative expression of the same expectation value

$$
\langle\cos (\phi)\rangle_{\beta}=\int_{-\pi}^{\pi} \frac{d \phi}{2 \pi I_{0}(\beta)} \cos (\phi) e^{\beta \cos (\phi)}=\frac{I_{1}(\beta)}{I_{0}(\beta)}=\left\langle e^{i \phi}\right\rangle_{\beta}
$$

has identical variance but different contour deformation possibilities

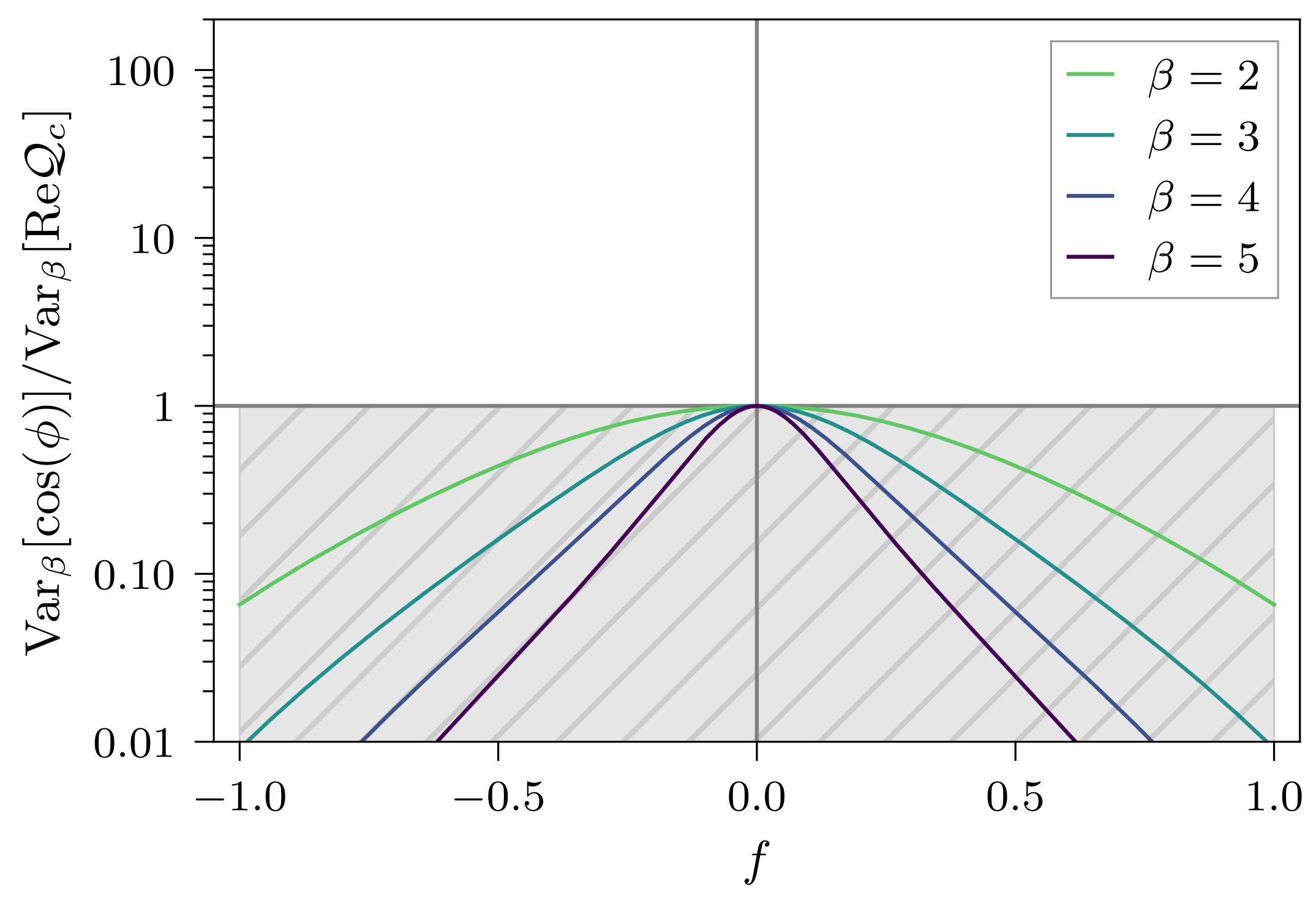

$\mathcal{Q}_{c}=\cos (\phi+i f) e^{\Delta S}$ 


\section{Parameterizing SU(2)}

Mean toy sign problem - SU(2) Wilson loops parameterized as

$$
\begin{aligned}
P & =\cos (\alpha / 2)+i \sin (\alpha / 2) \hat{n} \cdot \sigma \\
\frac{1}{2} \operatorname{Tr}(P) & =\cos (\alpha / 2)
\end{aligned}
$$

Useful to instead use angular parameterization

$$
P=\left(\begin{array}{cc}
\sin (\theta) e^{i \phi_{1}} & \cos (\theta) e^{i \phi_{2}} \\
-\cos (\theta) e^{-i \phi_{2}} & \sin (\theta) e^{i \phi_{1}}
\end{array}\right)
$$

Nice toy sign problem - $(1,1)$ component only

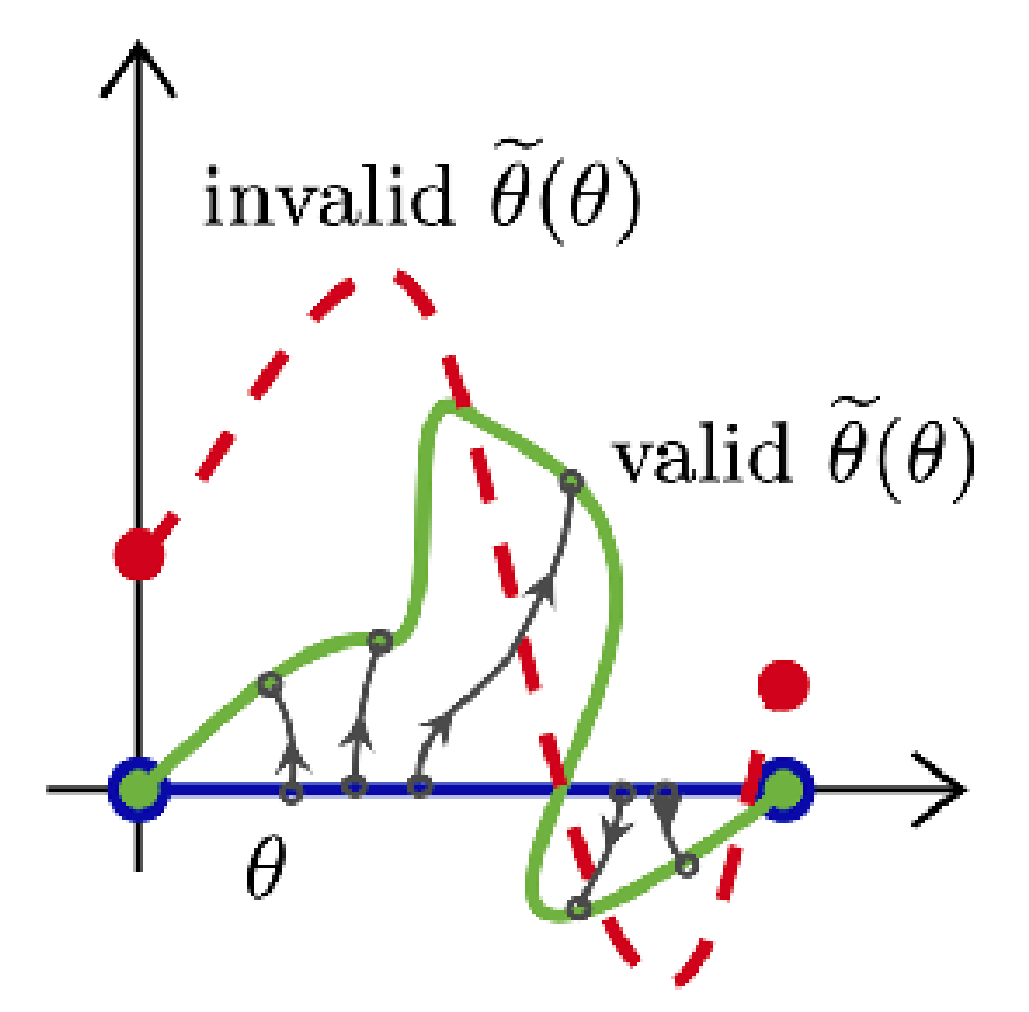

$$
\left\langle\frac{1}{2} \operatorname{Tr}(P)\right\rangle=\left\langle P_{11}\right\rangle=\left\langle\sin (\widetilde{\theta}) e^{i \widetilde{\phi}} e^{\Delta S} J(\theta, \phi)\right\rangle
$$




\section{Parameterizing SU(2) contours}

More general manifolds can be parameterized using gauge-fielddependent vertical deformations (ordered in spacetime)

$$
\widetilde{\Omega}=\Omega+i \sum_{\left\{n_{i}\right\}=0}^{\Lambda} \sum_{\left\{m_{j}\right\}=1}^{\Lambda} \lambda_{n_{1} \ldots m_{K}} \prod_{i=1}^{J} \sin \left(\phi_{i} n_{i}+\chi^{i}\right) \prod_{j=1}^{K} \sin \left(2 \theta_{j} m_{j}\right)
$$

Variance minimization is a wellposed optimization problem suitable for machine learning techniques

Overtraining on sample variance avoided using resampling (mini-batches) and using a separate test set for train rate scheduling

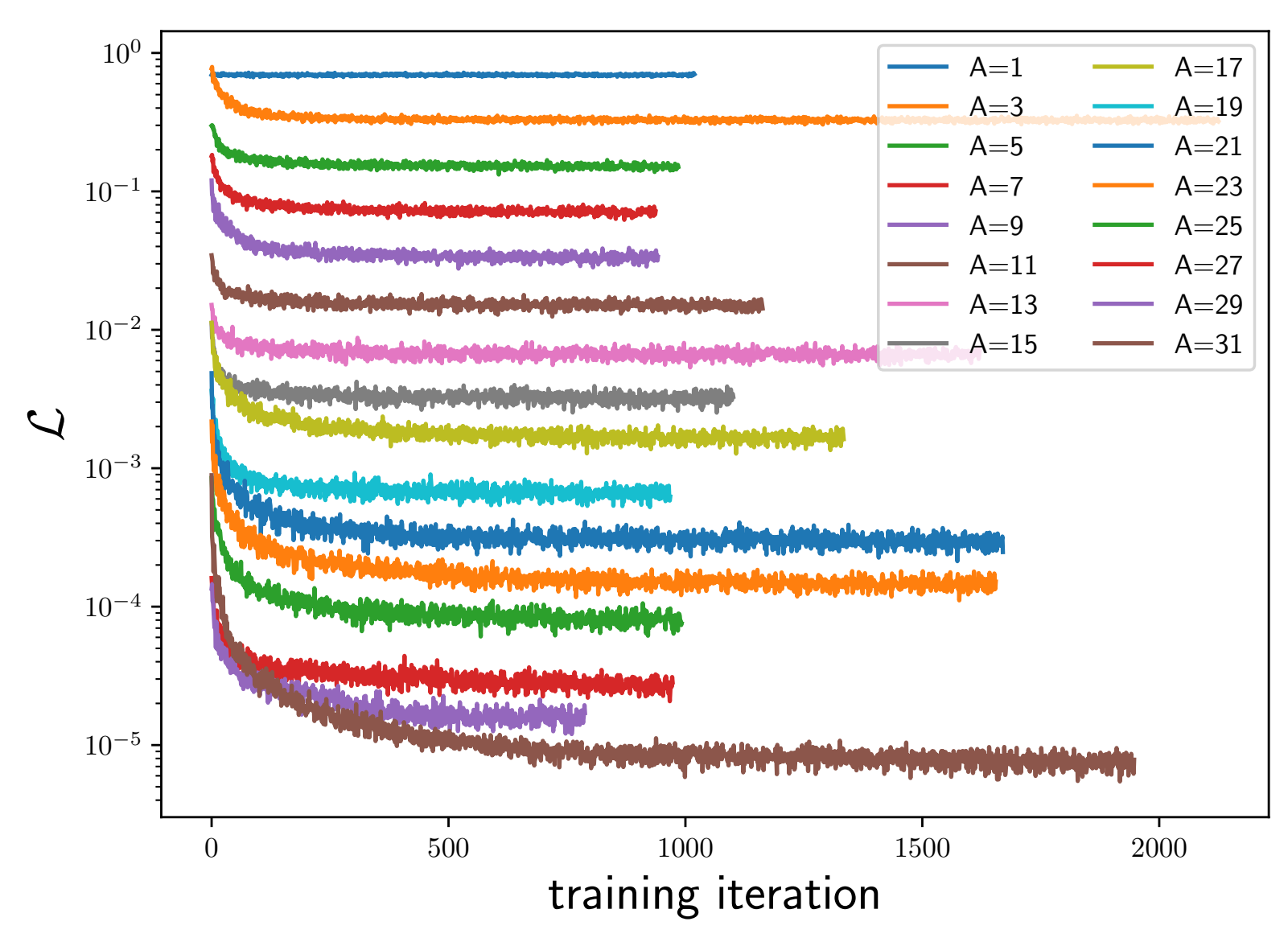




\section{SU(2) deformation results}

Optimized manifolds lead to exponentially increasing variance reduction for large area Wilson loops
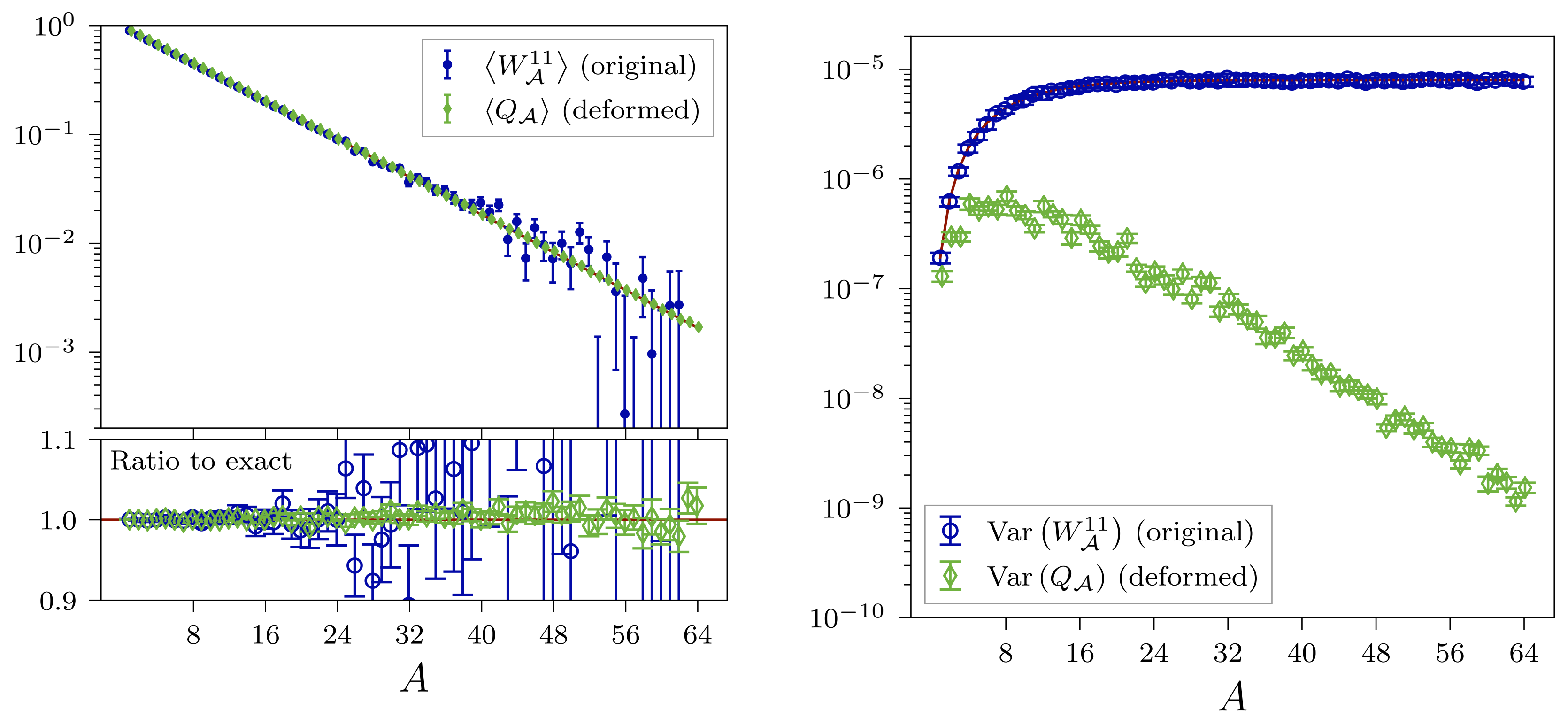


\section{SU(2) deformation results}

Variance reduction scales in physical units as gauge coupling is decreased towards continuum limit

Gains predominantly coming from zeroth order Fourier cutoff (constant vertical deformations)
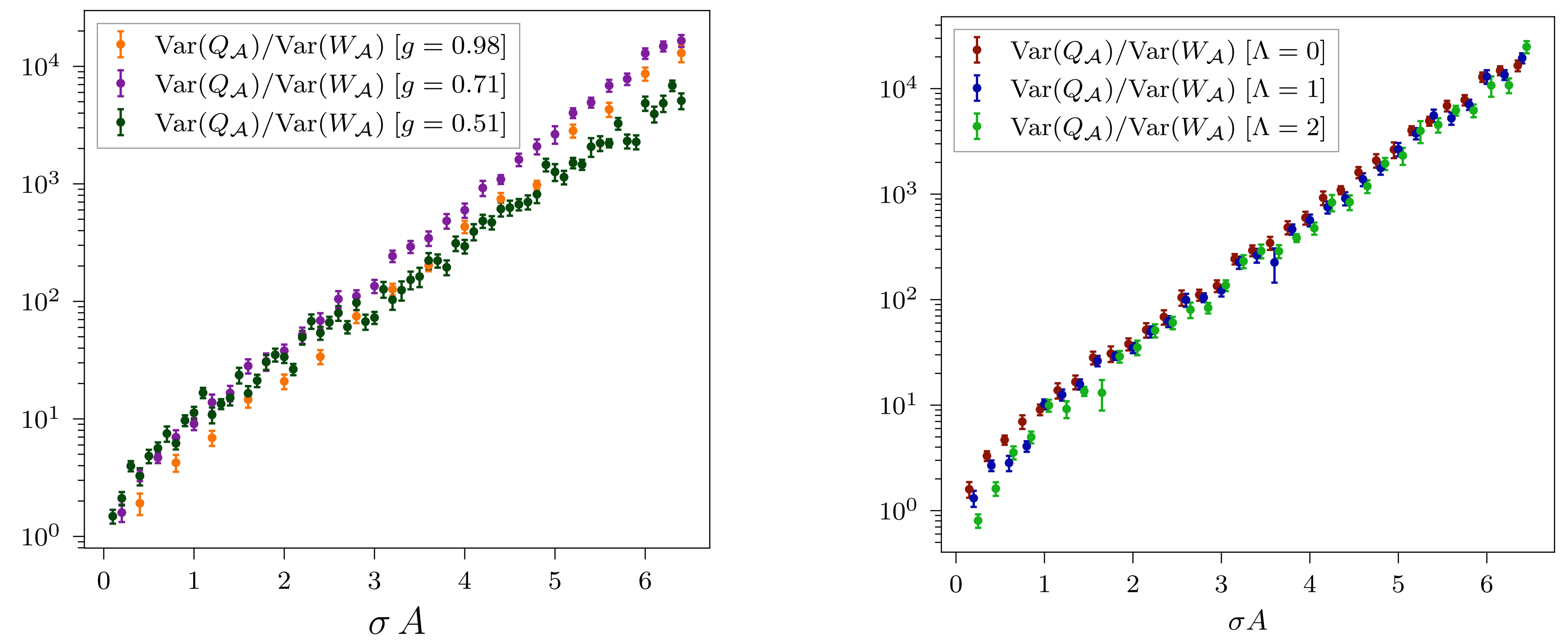


\section{$\mathrm{SU}(2)$ deformation results}

Optimal manifold resembles a constant magnitude deformation of diagonal phase throughout Wilson loop, linearly decreasing deformation of off-diagonal phase

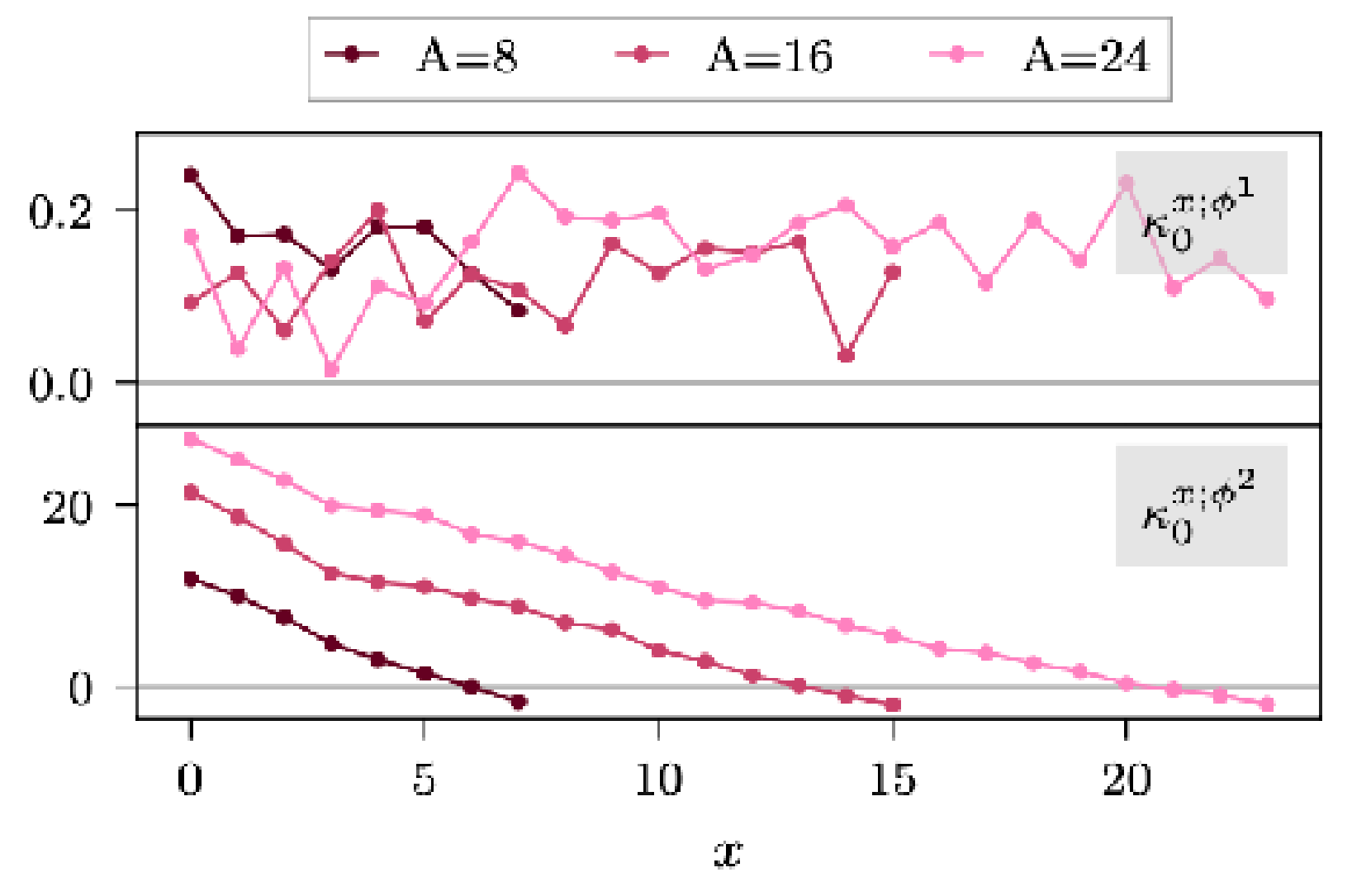

Qualitative features follow from minimizing magnitude of product of two plaquettes

$$
\begin{aligned}
\left(P_{x} P_{x^{\prime}}\right)^{11}= & \sin \theta_{x} \sin \theta_{x^{\prime}} e^{i \phi_{x}^{1}+i \phi_{x^{\prime}}^{1}} \\
& +\cos \theta_{x} \cos \theta_{x^{\prime}} e^{i \phi_{x}^{2}-i \phi_{x^{\prime}}^{2}}
\end{aligned}
$$




\section{SU(3) contour deformations}

\section{Analogous angular parameterization of SU(3) matrices}

$$
\phi_{x}^{1}, \ldots, \phi_{x}^{5}, \theta_{x}^{1}, \ldots, \theta_{x}^{3}
$$
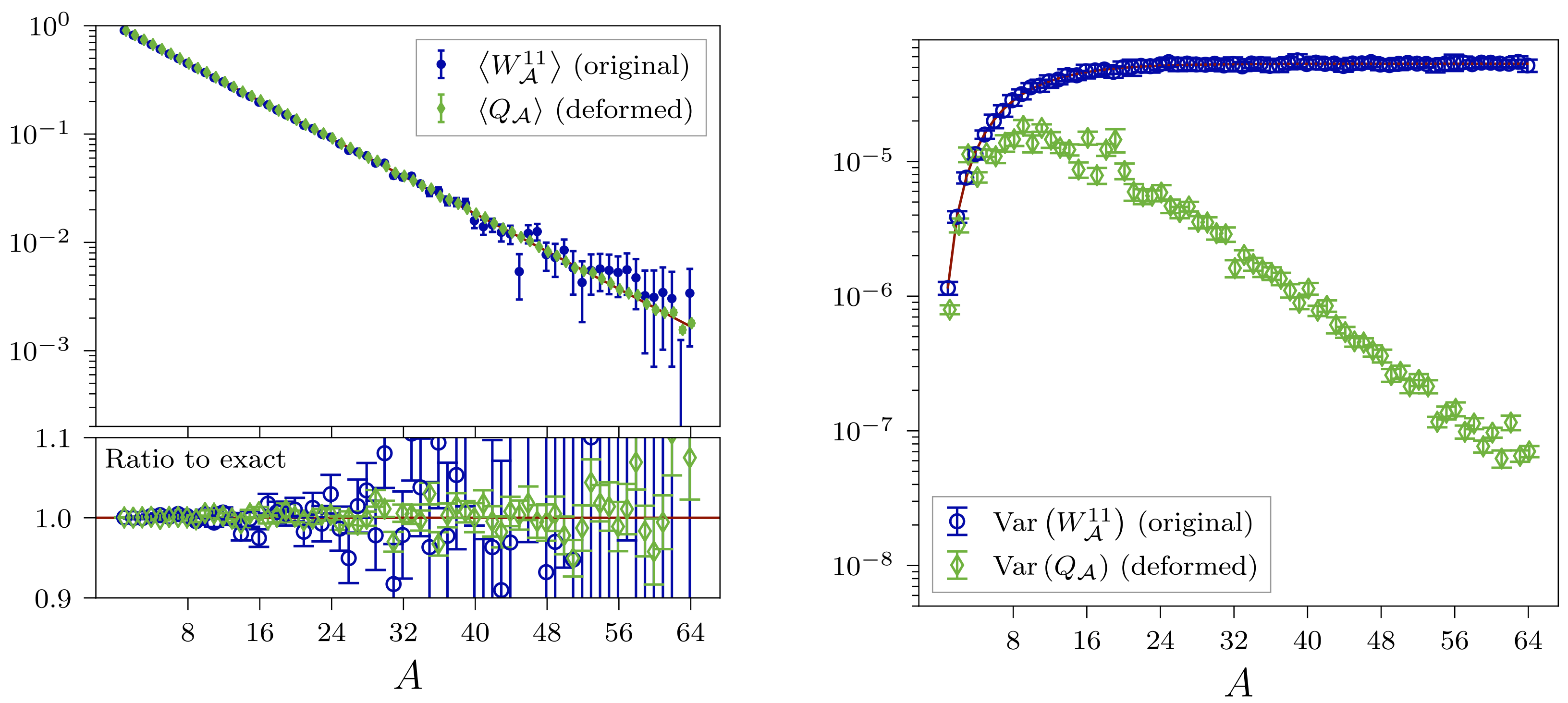

Detmold, Kanwar, Lamm, MW, Warrington, arXiv:2101.12668 


\section{$\mathrm{SU}(3)$ deformation results}

Variance reduction roughly scales in physical units as gauge coupling is decreased towards continuum limit (training?)

Gains predominantly coming from zeroth order Fourier cutoff, qualitatively consistent with loop magnitude minimization
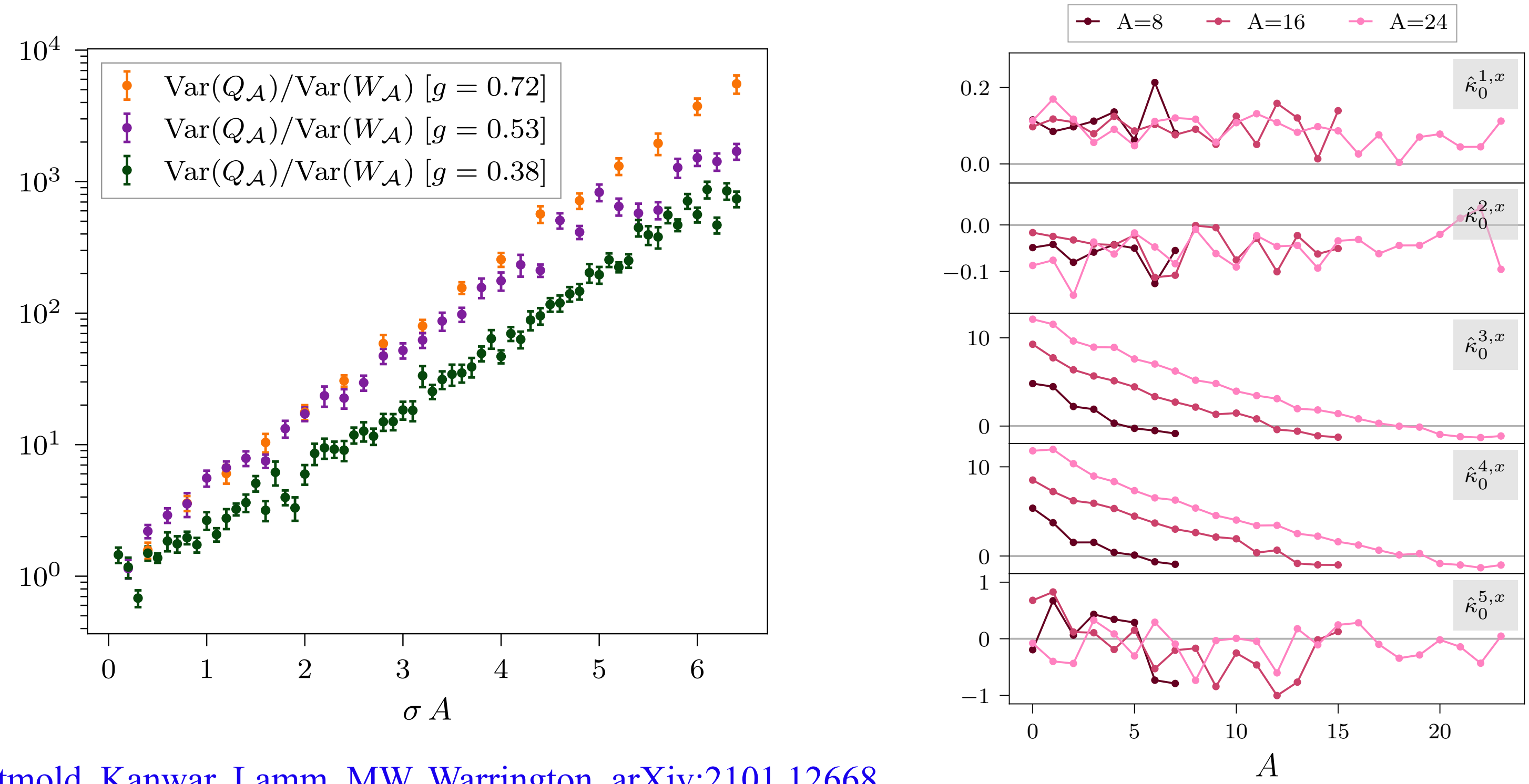


\section{Sign problem time}

Real-time evolution faces a notorious sign problem

$$
\langle\mathcal{O}\rangle_{M}=\frac{1}{Z_{M}} \int \mathcal{D} U e^{i S_{M}(U)} \mathcal{O}(U)
$$

Can trade sign for StN problem (compact gauge group) $\quad p(U) d U=d U$

$$
\langle\mathcal{O}\rangle_{M}=\frac{\int \mathcal{D} U p(U) \mathcal{O}(U) e^{i S_{M}(U)}}{\int \mathcal{D} U p(U) e^{i S_{M}(U)}}
$$

Still a problem, e.g. 2D U(1) $\operatorname{StN}\left(e^{i S_{M, U(1)}}\right) \approx \sqrt{n} e^{-2.3 V t}$

Improve with contour deformations $p(U) d U=e^{-\operatorname{Im}\left[S_{M}(\widetilde{U}(U))\right]}|J(U)| d U$

$$
\langle\mathcal{O}\rangle_{M}=\frac{\int \mathcal{D} U p(U) \mathcal{O}(U) e^{i \operatorname{Re}\left[S_{M}(\widetilde{U}(U))\right]+i \arg [J(U)]}}{\int \mathcal{D} U p(U) e^{i \operatorname{Re}\left[S_{M}(\widetilde{U}(U))\right]+i \arg [J(U)]}}
$$




\section{Real-time lattice gauge theory}

A more basic question: what's the action?

$$
S_{E, W}(U)=\frac{2}{g^{2}} \sum_{x} \sum_{\mu<\nu} \operatorname{Tr}\left(2-P_{x, \mu \nu}-P_{x, \mu \nu}^{-1}\right)
$$

Identifying time-space (space-space) plaquette terms as kinetic and potential energies and performing usual Wick rotation gives

$$
\begin{aligned}
S_{M, W}(U)=\frac{2}{g^{2}} \sum_{x} \sum_{k} \operatorname{Tr}\left(2-P_{x, 0 k}-P_{x, 0 k}^{-1}\right) \\
-\frac{2}{g^{2}} \sum_{x} \sum_{i<j} \operatorname{Tr}\left(2-P_{x, i j}-P_{x, i j}^{-1}\right)
\end{aligned}
$$




\section{(1+1)D Minkowski Wilson loops}

\section{Wick rotate action}

$$
g^{2} \rightarrow i g^{2} \quad\left\langle\frac{1}{2} \operatorname{Tr}\left(W_{\mathcal{A}}\right)\right\rangle_{M, W, S U(2)}=\left[\frac{I_{1}\left(4 i / g^{2}\right)}{I_{2}\left(4 i / g^{2}\right)}\right]^{-L t}
$$

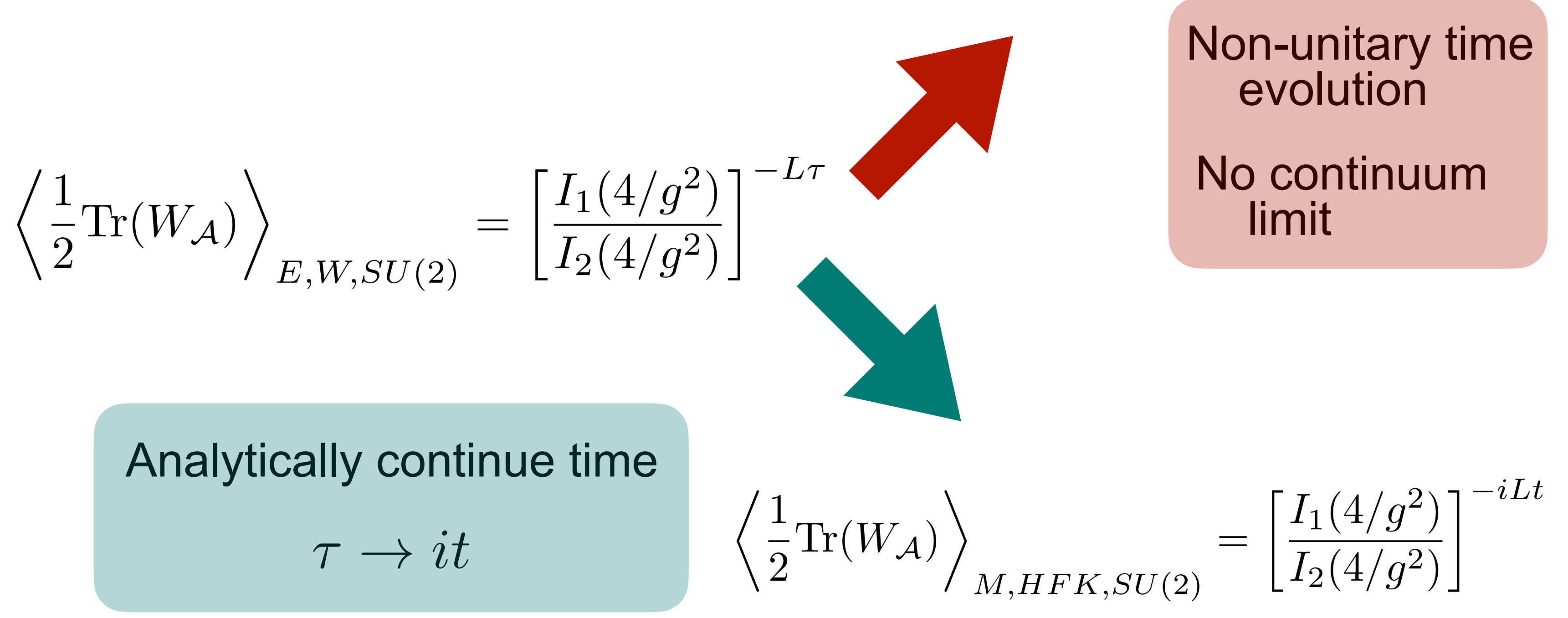




\section{The HFK action}

A unitarity time-evolution operator exists for the Wilson action

$$
\hat{T}=e^{-\hat{H}} \quad \hat{U}=e^{-i \hat{H}}=\hat{T}^{i}
$$

However, $\hat{U}$ is not the lattice time-evolution operator

An alternative action proposed by Hoshina, Fujii, and Kikukawa using character expansion leads to a unitary lattice timeevolution operator

$$
\begin{array}{r}
e^{i S_{M, H K F}(U)}=e^{-\frac{i}{e^{2}} \sum_{x} \sum_{i<j}\left(1-\cos \left(\phi_{x, i j}\right)\right)} \prod_{x, k}\left[\sum_{r=-\infty}^{\infty}\left[I_{r}\left(-i / e^{2}\right)\right]^{i} e^{i / e^{2}} e^{i r \phi_{x, 0 k}}\right] \\
P_{x, \mu \nu}=e^{i \phi_{x, \mu \nu}}
\end{array}
$$




\section{The heat-kernel action}

An alternative Euclidean action starts from "heat-kernel" equation

$$
\partial_{\tau} \mathcal{K}_{E}(U, \tau)=\Delta \mathcal{K}_{E}(U, \tau)
$$

Laplace-Beltrami operator for gauge group

Solution for $\mathrm{U}(1): \mathcal{K}_{E, U(1)}\left(e^{i \phi},-e^{2}\right)=\sum_{n=-\infty}^{\infty} \exp \left[-\frac{1}{2 e^{2}}(\phi+2 \pi n)^{2}\right]$

Generalization to $\mathrm{SU}(\mathrm{N})$ gives heat-kernel action

Analytic continuation gives Schrödinger equation

$$
i \partial_{t} \mathcal{K}_{M}(U, t)=-\Delta \mathcal{K}_{M}(U, t)
$$

Solution for $\mathrm{U}(1): \mathcal{K}_{M, U(1)}\left(e^{i \phi},-e^{2}\right)=\sum_{n=-\infty}^{\infty} \exp \left[\frac{i}{2 e^{2}}(\phi+2 \pi n)^{2}\right]$ 


\section{Euclidean U(1) heat-kernel results}

Infinite sum in Euclidean heat-kernel action can be performed stochastically by introducing integer-valued auxiliary field

$$
\int \mathcal{D} U e^{-S(U)}=\left(\int \mathcal{D} U \sum_{n=-\infty}^{\infty}\right) e^{-S(U, n)}
$$

Monte Carlo results for 2D Wilson loops with heat-kernel action with appropriately tuned coupling identical to Wilson action
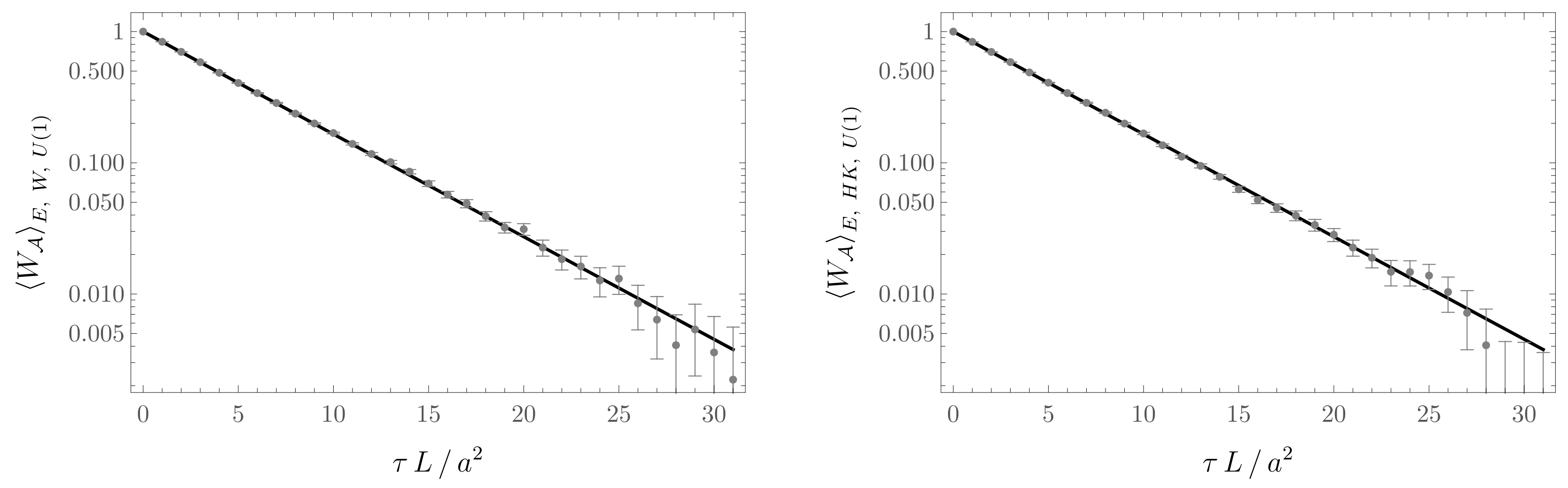


\section{Real-time U(1) lattice gauge theory}

In Minkowski spacetime, the sums defining both the HFK and heat-kernel actions diverge

Path integral contour deformations can improve convergence

$$
U \rightarrow \widetilde{U}(U, n)
$$

With the heat-kernel action, they also "solve" the sign problem

$$
e^{\frac{i}{2 e^{2}}(\phi+2 \pi n)^{2}} \rightarrow e^{-\frac{1}{2 e^{2}}(\phi+2 \pi n)^{2}}
$$
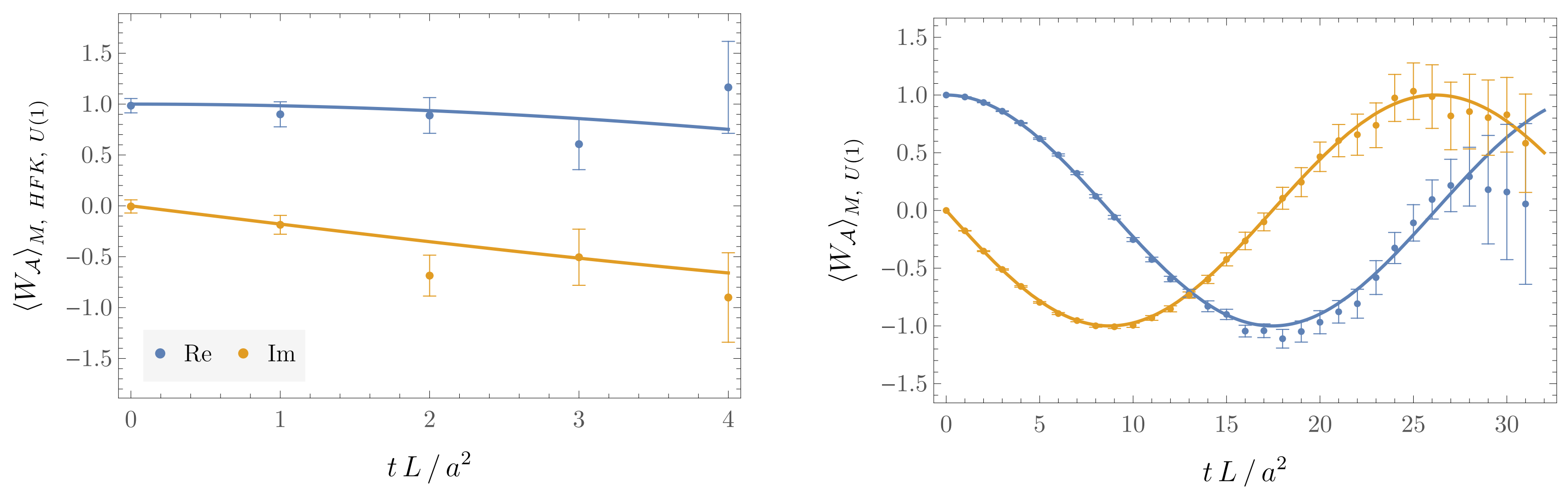


\section{Real-time SU(3)}

Works in $(1+1) D$

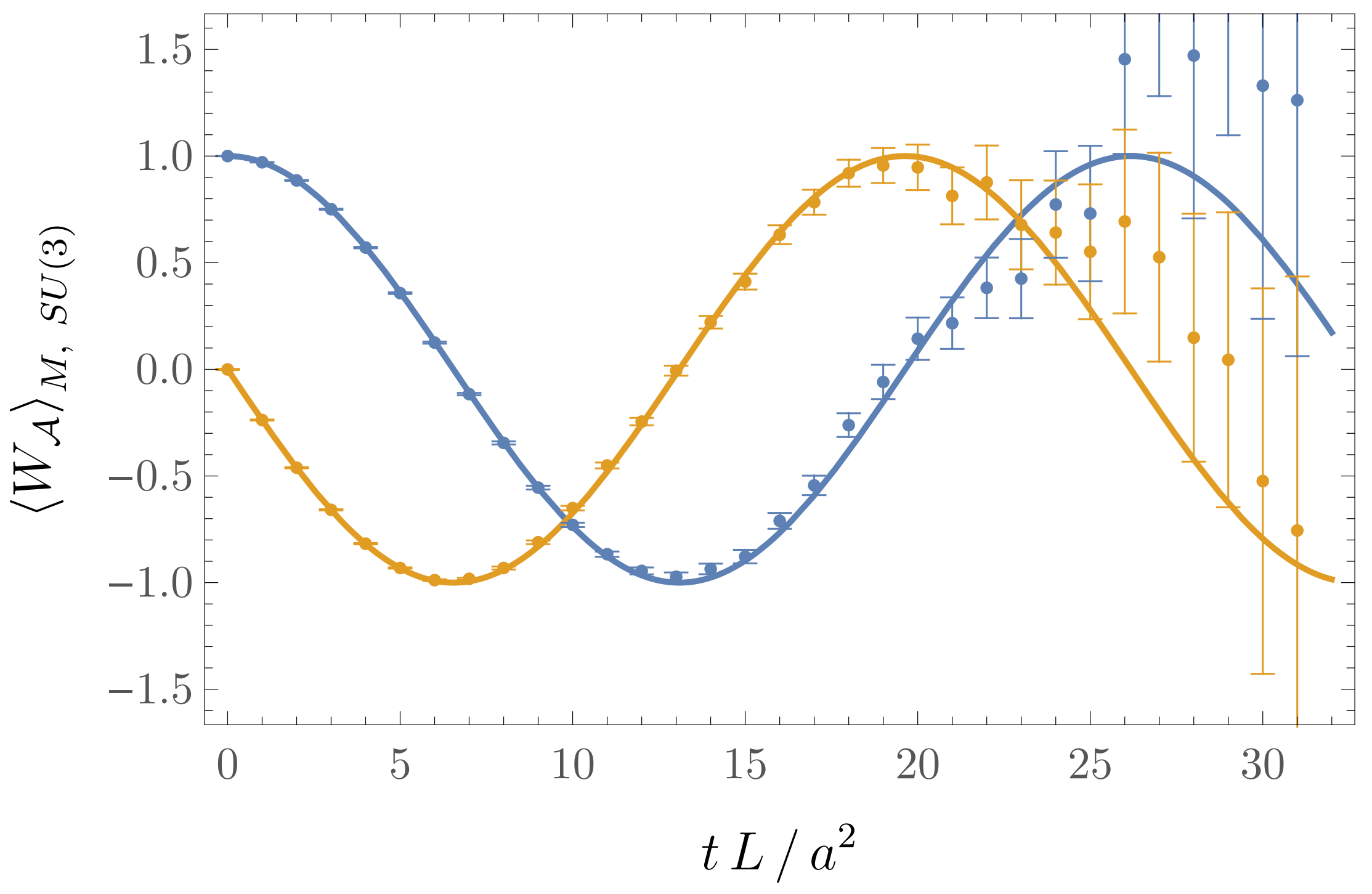




\section{Conclusions}

Lattice QCD is increasingly important for HEP/NP problems; sign(al-to-noise) problems present challenges
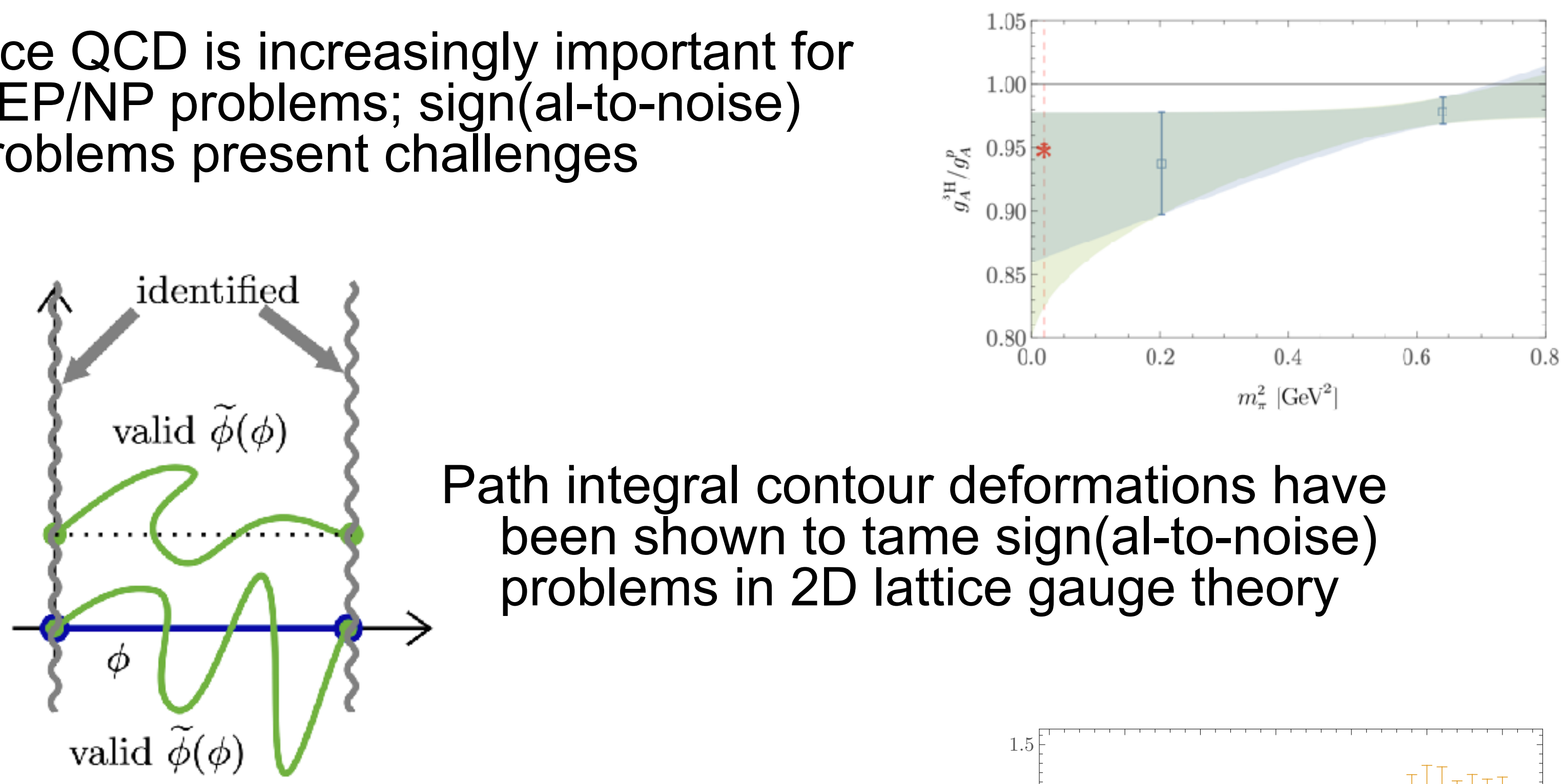

Path integral contour deformations have been shown to tame sign(al-to-noise) problems in $2 \mathrm{D}$ lattice gauge theory

Monte Carlo simulations of real-time lattice gauge theory are possible with the right action and contour deformation

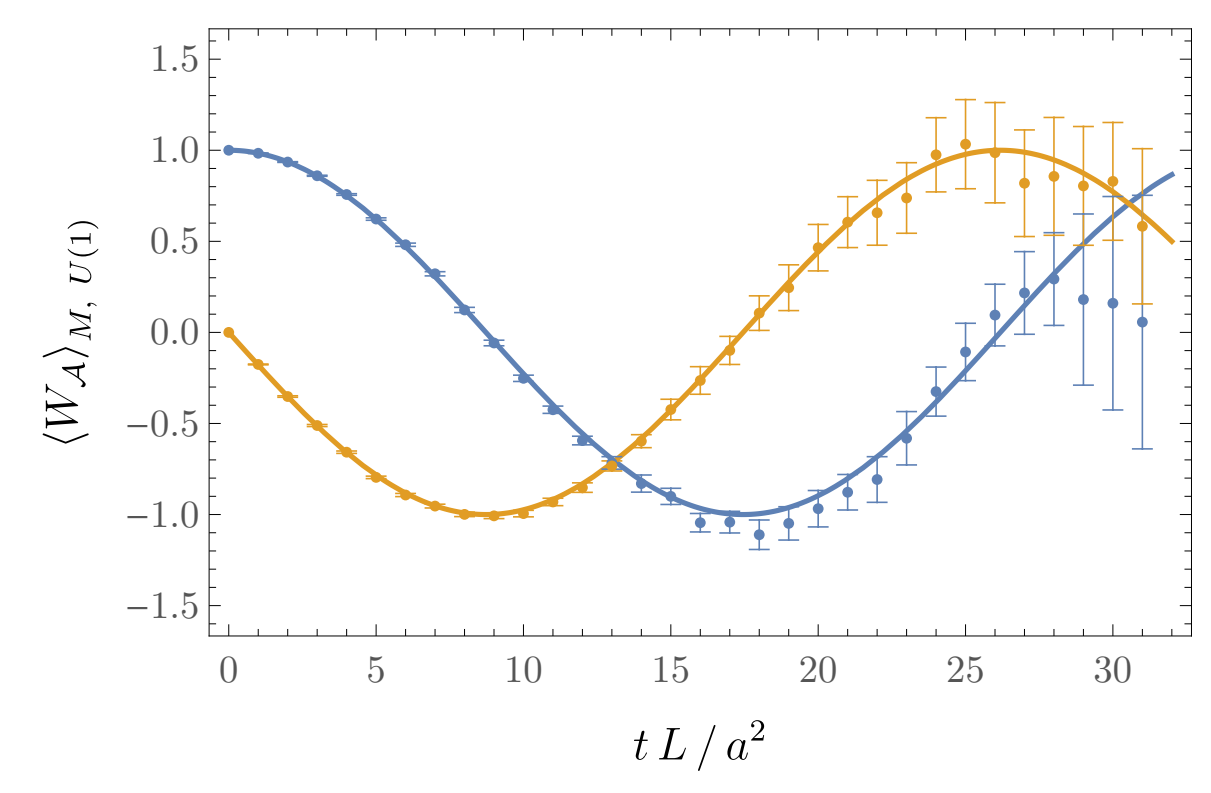

